\title{
Signals from the Superficial Layers of the Superior Colliculus Enable the Development of the Auditory Space Map in the Deeper Layers
}

\author{
Andrew J. King, Jan W. H. Schnupp, and lan D. Thompson \\ University Laboratory of Physiology, Oxford OX1 3PT, United Kingdom
}

We have examined whether the superficial layers of the superior colliculus (SC) provide the source of visual signals that guide the development of the auditory space map in the deeper layers. Anatomical tracing experiments with fluorescent microspheres revealed that a retinotopic map is present in the newborn ferret SC. Aspiration of the caudal region of the superficial layers of the right SC on postnatal day 0 did not cause a reorganization of this projection. Consequently, recordings made when the animals were mature showed that visual units in the remaining superficial layers in rostral SC had receptive fields that spanned a restricted region of anterior space. Auditory units recorded beneath the remaining superficial layers were tuned to corresponding anterior locations. Both the superficial layer visual map and the deeper layer auditory map were normal in the left, unoperated SC. The majority of auditory units recorded throughout the deeper layers ventral to the

The registration of sensory maps in the superior colliculus (SC) allows the different cues associated with multisensory objects to be integrated in ways that facilitate goal-directed orienting behavior (Stein and Meredith, 1993). Aligning these maps during development is an activity-dependent process that relies heavily on sensory experience. This applies particularly to the synthesis of a map of auditory space, which requires that SC neurons become tuned systematically to monaural and binaural localization cues that are initially encoded in separate brainstem pathways.

Several studies have highlighted the importance of visual experience in shaping the development of the map of auditory space. If the representation of visual space in the SC is shifted systematically by surgically deviating the eye in young ferrets (King et al., 1988) or by raising owls with prismatic spectacles that optically displace the visual field (Knudsen and Brainard, 1991), a corresponding change takes place in the auditory map. Consequently, the alignment between the preferred visual and auditory stimulus locations is preserved. On the other hand, various abnormalities in the auditory representation have been reported in animals that have been deprived of visual cues during develop-

\footnotetext{
Received June 23, 1998; revised Aug. 28, 1998; accepted Sept. 2, 1998.

We are grateful to the Wellcome Trust and the EU Biotechnology Program (Bio4CT960000) for financial support and to Patricia Cordery for excellent histological assistance. A.J.K. holds a Wellcome Senior Research Fellowship and J.W.H.S. holds a Dunhill Research Fellowship (awarded by Defeating Deafness: the Hearing Research Trust).

Correspondence should be addressed to Dr. Andrew J. King, University Laboratory of Physiology, Parks Road, Oxford OX1 3PT, UK.

Copyright (C) 1998 Society for Neuroscience $\quad 0270-6474 / 98 / 189394-15 \$ 05.00 / 0$
}

superficial layer lesion were also tuned to single sound directions. In this region of the SC, however, we observed much greater scatter in the distribution of preferred sound directions and a significant increase in the proportion of units with spatially ambiguous responses. The auditory representation was degraded, although many of these units were also visually responsive. Equivalent lesions of the superficial layers made in adult ferrets did not alter the topographic order in the auditory representation, suggesting that visual activity in these layers may be involved in aligning the different sensory maps in the developing SC.

Key words: sound localization; superior colliculus; auditory space map; visual space map; bimodal neurons; ferret; developmental plasticity; retinocollicular projection; superficial layers; lesion ment (Withington-Wray et al., 1990; Knudsen et al., 1991; Withington, 1992; King and Carlile, 1993).

The nature of the process by which visual signals calibrate the developing auditory responses is unknown. In prism-reared owls, changes in the auditory map are brought about by retuning neurons to interaural time differences (ITD) that correspond to the optically displaced visual map. These shifts in ITD tuning are first observed in the external nucleus of the inferior colliculus (ICX) (Brainard and Knudsen, 1993), which projects topographically to the SC (Knudsen and Knudsen, 1983). Paradoxically, the interaction between the two modalities, therefore, takes place in barn owls in what appears to be a purely auditory nucleus, rather than at the level of the bimodal neurons in the SC.

Although most neurons throughout the barn owl SC are bimodal (Knudsen, 1982), the superficial layers of the mammalian SC are exclusively visual in function. Anatomical (Grantyn et al., 1984; Mooney et al., 1984, 1988; Moschovakis et al., 1988; Behan and Appell, 1992; Hall and Lee, 1997) and physiological (Mooney et al., 1992; Lee et al., 1997) studies have revealed the existence of columnar connections between the superficial visual and the deeper multisensory layers. Moreover, in ferrets, the superficial layers project topographically to the nucleus of the brachium of the inferior colliculus (BIN) (Baron et al., 1998), the primary source of auditory input to the deep SC (Schnupp and King, 1997).

These studies suggest that, in mammals, the superficial SC may be part of the circuit by which visual signals influence the maturation of auditory spatial tuning. If so, partial removal of this region early in development should result in a localized change in the underlying auditory map that is comparable to the effects of 
visual deprivation. To test this possibility, we have examined the effects on the visual and auditory representations of making restricted lesions of the superficial layers in both neonatal and adult ferrets.

\section{MATERIALS AND METHODS}

Superior colliculus lesions. A partial lesion of the superficial layers of the SC was made unilaterally in nine ferret kits from two different litters on the day of birth (P0). The animals were anesthetized with Saffan (Alfaxalone/Alfadolone acetate; Mallinckrodt Veterinary Ltd., Uxbridge, $\mathrm{UK}$; dose equivalent to $0.6-0.8 \mathrm{ml} / \mathrm{kg}$ ) and the midbrain exposed by removal of the overlying cranium and dura. At this age, the cortex does not overly the SC. The superficial layers of the right SC were sectioned mediolaterally, and the caudal region was removed by aspiration. Care was taken to avoid the adjacent inferior colliculus. At the conclusion of the surgery the animals were recovered and returned to their mothers.

Similar lesions were also made in the right SC of two adult ferrets (both just over 1 year in age) that were anesthetized with Saffan (2 $\mathrm{ml} / \mathrm{kg}$ ). The scalp was reflected, the cranium over the SC was removed, and the cortex was aspirated unilaterally to expose the right SC. Once the superficial layer ablation had been made, the brain cavity was packed with Sterispon (Allen and Hanbury, London, UK), the cranium was replaced, and the scalp was sutured. The animals received antibiotic (Penbritin; SmithKline Beecham, Brentford, UK; $0.2 \mathrm{ml}$, i.m.) and opiate analgesia (Temgesic; Reckitt and Colman, Hull, UK; 0.05 ml, i.m.).

In each case, the extent of the lesion was determined both physiologically by recording visual responses in the remaining superficial layers and/or histologically when the animals were used in a terminal tract tracing or recording experiment.

Anatomical tracing experiments. The state of the retinocollicular projection was analyzed in a further nine newborn ferrets. P0 ferret kits were anesthetized either with ether or with Saffan, the scalp was reflected, and the cranium and dura over the SC were removed. In the initial experiments, multiple injections of either red or green fluorescent microspheres (LumaFluor, Naples, FL; diluted 1:10 with sterile saline) were made in caudal SC; usually, a single injection of the other tracer was made in rostral SC. In a subsequent group of animals, a single injection of one tracer was made in caudal SC, and a single injection of the other tracer was made in rostral SC; the separation of these paired injections was systematically varied. The volume of the single injections was $50 \mathrm{nl}$, and for the multiple injections a total volume of 100-200 $\mathrm{nl}$ was injected. The cranium was replaced, the scalp was sutured, and the animals survived overnight in an incubator. After a terminal dose of pentobarbitone sodium (Sagatal; Rhône Mérieux, Harlow, UK; $120 \mathrm{mg} / \mathrm{kg}$, i.p.), the kits were perfused through the heart with PBS, followed by a brief perfusion with $1 \%$ paraformaldehyde in phosphate buffer. The superior pole of the cornea was marked with a knife cut, the eyes were removed, and the retinas were dissected out, post-fixed with $4 \%$ paraformaldehyde in phosphate buffer, and prepared as a flat mount. After removing the eyes, the perfusion continued with $4 \%$ paraformaldehyde in phosphate buffer. The brain was post-fixed and $30 \mu \mathrm{m}$ frozen sections were cut to allow reconstruction of the injection sites.

Analysis of the distribution of labeled ganglion cells was performed with a computer-microscope package, using a camera lucida to superimpose the mouse-drawn images over the image of the retina. The retinas were sampled at regular intervals (either at 500 or $200 \mu \mathrm{m}$ ), and all the labeled cells in a sample box were counted (sample box size either $125 \times$ $125 \mu \mathrm{m}$ or $75 \times 75 \mu \mathrm{m}$, respectively). Cells of one color were drawn, the screen was cleared, and then cells of the other color were drawn. The software identified candidate cells as double-labeled; these were then confirmed visually. Isodensity contours were drawn using Systat (Version 5.0 for Windows) and expressed as a percentage of the maximum number of cells of a given color found in a sample box in that retina. A graphics tablet was used to measure the retinal area bounded by the $20 \%$ and by the $40 \%$ contour lines; these areas were expressed as a percentage of the total retinal area. The segregation of the cells was also measured with a nearest neighbor analysis (Krug et al., 1998). Briefly, the program determined whether the nearest neighbor of a given cell was labeled with the same or with a different tracer. A value of 1 was given if the neighbor was the same color and a value of 0 if different (if the cell was double-labeled, a value of 0.5 was used). Given equally sized populations of red and green cells, totally segregated populations will have an average neighbor value of $100 \%$, and totally intermixed populations will have an average of $50 \%$. Simulations have shown that changes in the relative sizes of the two populations cause the mean values for each population to deviate symmetrically away from the values found with matched populations, provided the ratio of population numbers is not $>4: 1$ (Krug et al., 1998). Consequently, we have averaged the two neighbor values (one for red cells and one for green cells) obtained on each retina and have excluded retinas in which the ratio of the two populations exceeded 4:1.

The state of the retinal projection was analyzed in three of the adult ferrets ( $>6$ months of age) that had undergone partial ablation of the SC at $\mathrm{P} 0$ using retrograde tracing of horseradish peroxidase (HRP). The animals were anesthetized with Saffan $(2 \mathrm{ml} / \mathrm{kg}$, i.m.). The scalp was reflected, the cranium over the SC was removed, and the cortex was aspirated to reveal the SC. Multiple injections of HRP (20\% in 2\% DMSO in saline; total volume of $4 \mu \mathrm{l}$ ) were made into the remaining rostral portions of the SC. The injections avoided the more medial portion of the SC to minimize spread across the midline. The brain cavity was packed with Sterispon, the cranium was replaced, and the scalp was sutured. The animals received injections of Penbritin $(0.2 \mathrm{ml}$, i.m.) and Temgesic $(0.05 \mathrm{ml}$, i.m. $)$ and survived for $2-3 \mathrm{~d}$. After a terminal dose of Sagatal $(120 \mathrm{mg} / \mathrm{kg}$, i.p.), the animals were perfused through the heart with PBS followed by a brief perfusion with $1 \%$ paraformaldehyde in phosphate buffer. The superior pole of the cornea was marked with a knife cut, the contralateral eye was removed, and the retina was dissected out, prepared as a flat mount, and post-fixed for $2 \mathrm{hr}$ with $1 \%$ paraformaldehyde and $1.25 \%$ glutaraldehyde in phosphate buffer. After removing the eye, the perfusion continued with $1 \%$ paraformaldehyde and $1.25 \%$ glutaraldehyde in phosphate buffer. The retinas were reacted for HRP using a modified Hanker-Yates protocol (Perry and Linden, 1982). The distribution of labeled ganglion cells was analyzed using the computer microscope, with a sample interval of $500 \mu \mathrm{m}$ and a sample box size of $125 \times 125 \mu \mathrm{m}$.

Forty-eight hours before the terminal recording experiment, another three of the ferrets that had received lesions of the SC at P0 were reanesthetized with Saffan and $500 \mu \mathrm{Ci}$ of $\left[{ }^{3} \mathrm{H}\right]$ proline (Amersham) were injected into the vitreous humor of the left eye. The post-fixed brains were cut parasagittally at 30 or $40 \mu \mathrm{m}$. Air-dried sections were dehydrated, defatted, and then coated with nuclear research emulsion. The sections were exposed at $4^{\circ} \mathrm{C}$ for $4-8$ weeks and then developed. The developed sections were counter-stained with cresyl violet and coverslipped.

Electrophysiological recording. Full details are given in King and Hutchings (1987) and Schnupp et al. (1995). Six of the ferrets in which a partial lesion of the superficial layers had been made on P0 were prepared for electrophysiological recording when they were at least 7 months old. Recordings were also made from the two ferrets that had received caudal SC ablations in adulthood, beginning 105 and $111 \mathrm{~d}$ after surgery, respectively. Anesthesia was induced with Saffan and maintained during surgery by supplementary intravenous doses. Body temperature was maintained at $\sim 39^{\circ} \mathrm{C}$. A craniotomy was made bilaterally above the cortex overlying the SC and a minimal metal headholder, which surrounded both craniotomies and which supported the animal from behind, was attached to the skull. The eyelids were trimmed, the pupils were dilated with atropine sulfate, and the eyes were protected with zero refractive power contact lenses to allow mapping of visual receptive fields. To prevent eye movements, the ferrets were paralyzed with Flaxedil (gallamine triethiodide; Sigma, St. Louis, MO; $12 \mathrm{mg}$, i.v.). Anesthesia and paralysis were maintained with a continuous intravenous infusion of Nembutal (pentobarbitone sodium; Abbott Laboratories, Queensborough, UK; $\left.1 \mathrm{mg} \cdot \mathrm{kg}^{-1} \cdot \mathrm{hr}^{-1}\right)$ and Flaxedil (20 $\mathrm{mg} \cdot \mathrm{kg}^{-1} \cdot \mathrm{hr}^{-1}$ ), respectively. The ferrets were ventilated artificially with room air supplemented with $95 \% \mathrm{O}_{2}$ and $5 \% \mathrm{CO}_{2}$, and the heart rate, electrocardiogram, electroencephalogram, and end-tidal $\mathrm{CO}_{2}$ were monitored continuously.

All recordings were performed in an anechoic chamber. The animal was placed on a small table at the center of a vertical robotic hoop with a radius of $0.65 \mathrm{~m}$ (Annetts et al., 1987). A Kef T27 loudspeaker and $1 \mathrm{~cm}$ diameter LED were mounted on the hoop. Movement of the speakerLED assembly along the hoop allowed the vertical angle of the stimulus to be varied, whereas the azimuthal angle was varied by rotating the hoop around the animal. These movements were directed either by a remote control unit or by the data acquisition software.

Neural activity was recorded with a tungsten microelectrode that was lowered vertically through the intact cortex and into the SC using a remotely controlled, motorized microdrive. The neural signals were amplified $(\sim 10,000$ times), bandpass filtered $(500-5000 \mathrm{~Hz})$, and digitized $(25 \mathrm{kHz}, 8$ bit). Single units were isolated using our own spike- 
sorting software (Schnupp et al., 1995; Schnupp and King, 1997). We typically isolated three single units at each recording site.

As the electrode was lowered vertically through the cortex, $100 \mathrm{msec}$ light flashes were presented at a rate of $\sim 0.8 \mathrm{~Hz}$ from a 1 -cm-diameter LED mounted in front of the contralateral eye. The appearance of a strong, characteristic response evoked by this flashing, stationary stimulus indicated that the electrode had entered the superficial layers of the SC. We then used the hoop-mounted LED to map the receptive fields of both single units and multiunits in the superficial layers. Subsequently, the electrode was advanced into the deeper layers of the SC as auditory stimuli, consisting of $100 \mathrm{msec}$ broadband noise bursts $(30-30,000 \mathrm{~Hz}$, $100 \mathrm{msec}$ duration with a $5 \mathrm{msec}$ rise/fall time), were delivered with an interstimulus interval of $\sim 1.5 \mathrm{sec}$ from the hoop-mounted speaker. In those tracks in which the superficial layers were missing, no strong visual drive was encountered. The electrode was therefore advanced with both noise bursts and visual stimuli presented from within the contralateral hemifield.

Once an acoustically responsive unit was isolated, we estimated its threshold with the loudspeaker positioned near the receptive field center of the visual responses obtained in the superficial layers of the same electrode track or, if no superficial layer visual responses were encountered, at the angle from which the strongest auditory response was elicited for previous units in the track. To obtain the threshold, the sound level was reduced in 5 or $10 \mathrm{~dB}$ steps until the $95 \%$ confidence interval for the number of evoked spikes overlapped the baseline of spontaneous activity and a clear peak in the peristimulus time histogram (PSTH) was no longer apparent. We then determined the spatial response profile of each unit by measuring the response in $20^{\circ}$ azimuth steps from positions $160^{\circ}$ contralateral to $160^{\circ}$ ipsilateral to the recording site. The vertical coordinates of the speaker were held constant at the elevation of the visual receptive field center for the overlying superficial layers. For some of the electrode penetrations in which no superficial layer visual activity was encountered, we also measured auditory elevation profiles to ensure that the loudspeaker was positioned at an optimum angle for mapping the azimuth profile. All auditory responses were mapped at a sound level of 20-30 dB above unit threshold. Because the largest interaural level differences generated by the ferret's head are $\sim 25$ dB (Carlile, 1990; Carlile and King, 1994), this stimulus level will provide binaural cues at all frequencies and positions.

The discharge of the unit was measured in response to 20 stimulus presentations at each speaker position. After the first spatial profile was obtained we often redetermined the auditory unit threshold at the speaker position that gave the strongest response. The duration of the response was estimated by inspection of the PSTH pooled over all the data for each unit. This was used to set a window within which spikes were counted to measure the strength of the response. The period from $500-1000 \mathrm{msec}$ after stimulus onset, well after the end of the stimulusevoked response, was used to derive an estimate of the spontaneous activity of each unit. The number of stimulus-evoked spikes was calculated as the mean number of spikes per presentation in the response period minus the number of spikes during an equivalent length of time in the spontaneous period. Azimuth profiles were classified objectively by the data acquisition software into one of five categories: "tuned", "bilobed", "broad", "complex", or "omnidirectional". Tuned profiles contained a single peak, whereas bilobed profiles had two peaks, and complex cells had more than two. A peak in the response profile was defined as a region of at least $80 \%$ of the maximal response, flanked by regions with $\leq 40 \%$ of the maximal response, with the flanking regions not $>160^{\circ}$ apart. Broad cells exhibited a response peak that was wider than $160^{\circ}$; whereas omnidirectional responses remained above $40 \%$ of the maximum at all speaker positions tested. These same classification criteria are used routinely in this laboratory and, therefore, allow a direct comparison of the results reported here with those from previous studies.

Histological reconstructions of recording sites. Recording sites were marked with small electrolytic lesions ( $-5 \mu \mathrm{A}$ for $5 \mathrm{sec})$. At the end of the recording session, typically after $\sim 40 \mathrm{hr}$ of recording, the animal was overdosed with Sagatal and perfused through the heart with PBS followed by $10 \%$ formal saline. The brainstem was removed, cryoprotected with $30 \%$ sucrose, and cut into $50 \mu \mathrm{m}$ coronal sections on a freezing microtome. The Nissl-stained sections were used to assess the extent of the neonatal lesion and to reconstruct the recording sites. The histological coordinates of each recording site were expressed as a fraction of SC length and width and then plotted on a standardized SC template. The rostrocaudal coordinates of the recording sites were measured from the rostrolateral border on this standardized template in a direction normal to the isoazimuth contours previously described in normal, adult ferrets (King and Hutchings, 1987).

\section{RESULTS}

\section{Retinocollicular topography at PO}

With one exception, all retinas of animals injected at $\mathrm{P} 0$ gave a clear segregation of the red- and green-labeled populations of ganglion cells after injections of label into the SC. Figure 1 illustrates the distributions of labeled ganglion cells in three retinas taken from animals in which the separation of paired injections of tracer was varied systematically. In each of these animals, the green injection was placed caudally and slightly laterally, and the red injection was placed more rostrally; the separations give the distances between the centers of the injection sites. The dashed lines indicate isodensity contours for the redand the green-labeled populations. In each case, the outer contour encloses the area of retina where the density of labeled ganglion cells was at least $20 \%$ of the peak density found for that color. The retina in Figure $1 A$ displays the least segregation of any retina in the series with considerable overlap in the contours for the red and green populations. In fact, $>50 \%$ of the cells in the red focus, the smaller population, were double-labeled. The pattern of retinal labeling was not caused by overlapping injection sites; although only $240 \mu \mathrm{m}$ separated the injections, the diameter of the green injection site was $100 \mu \mathrm{m}$, and that of the red site was $70 \mu \mathrm{m}$. Despite the lack of segregation of red and green cells, the isodensity contour revealed that the greatest density of red cells was temporal to the center of the green population, as expected from the adult map. With increasing injection site separation, the segregation of the foci of red- and green-labeled ganglion cells increased (Fig. 1B,C), and the number of double-labeled cells decreased. No double labeling was found in the retina shown in Figure $1 C$, which is from the animal with the most widely separated injection sites.

The isodensity contour analysis reveals clear topography in the retinocollicular projection of the newborn ferret. The projection is not a strict point-to-point mapping, because there is both convergence and divergence in the projection. Thus, two points $240 \mu \mathrm{m}$ apart in the SC receive input from substantially overlapping retinal areas (Fig. $1 A$ ), and the axons of many retinal ganglion cells contact both sites. One indication of the retinal convergence is that the average injection site (in animals receiving only single injections of any one tracer) occupied just over $1 \%$ of the area of the SC, whereas the average percentage of retina labeled at $20 \%$ of peak density was $14 \%$; this value drops to $6.5 \%$ if the $40 \%$ isodensity contours are used. Another measure of topographic precision is the nearest neighbor value. Figure 2 illustrates how this changes with separation of the injection sites, showing increasing segregation with separation. The nearest neighbor value measures the scatter in the total populations of labeled cells and so is sensitive to the numbers of labeled ganglion cells distributed across the retina that are not shown by the isodensity contours. On average, $\sim 20 \%$ (mean $=21.7 \%$; SD $=$ $12.4 ; n=8$ ) of the labeled cells in a given retina fell outside the $20 \%$ density contour line. Thus, even when the injection sites were separated by $1 \mathrm{~mm}$ and the isodensity contours were clearly segregated (Fig. $1 C$ ), the neighbor value was still $<100 \%$. Because this halo of labeled cells scattered across the retina is not seen after injections in the adult SC (Wingate et al., 1992; I. D. Thompson, unpublished observations), these cells are presumably eliminated by ganglion cell death in the neonate. 

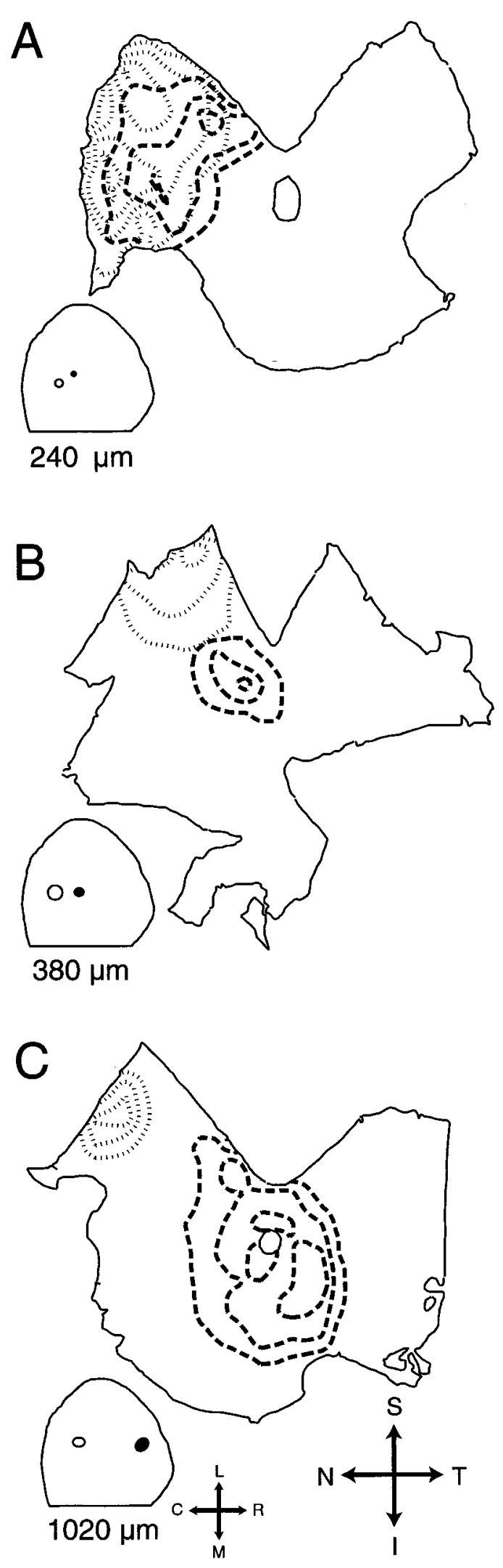

Figure 1. Retinocollicular topography in newborn ferrets. Flattened whole mounts of the contralateral retina showing the distribution of retrogradely labeled ganglion cells after a $50 \mathrm{nl}$ injection of green fluorescent beads into the caudolateral pole of the contralateral SC and an injection of red fluorescent beads into the more rostral SC in three different $\mathrm{P} 0$ ferrets. The insets to the bottom left of each panel represent

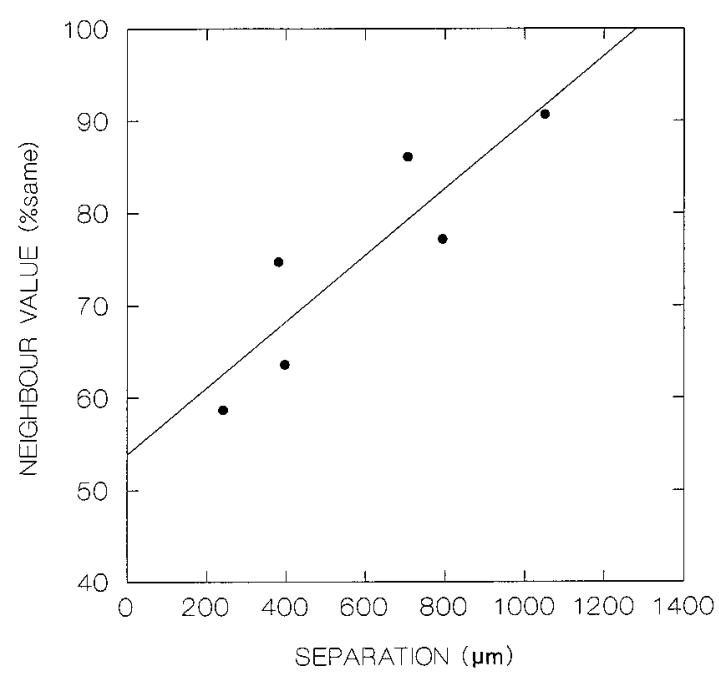

Figure 2. Nearest neighbor analysis of the retinocollicular topography in P0 ferrets. For a given retina, we estimated the probabilities (1) that any green cell had as its nearest neighbor another green cell and (2) that any red cell had as its nearest neighbor another red cell. These two probabilities were averaged to give a mean neighbor value for each retina (a value of $100 \%$ indicates two totally segregated populations, and a value of $50 \%$ indicates two totally overlapping populations). All the retinas analyzed for this figure were taken from ferrets that had a single injection of tracer in more rostral SC, and the separations indicate the distance between the center of the rostral injection and the center of the more caudal injection (in 3 cases, multiple caudal injections were made that were spaced mediolaterally; the separation gives the closest site). The graph shows how increasing the separation of the injections produces increasingly segregated populations of labeled retinal ganglion cells. However, even with a separation of $>1 \mathrm{~mm}$, the neighbor value does not reach $100 \%$ in $\mathrm{P} 0$ ferrets.

\section{Effect of neonatal superficial layer lesions on the representation of visual space}

The volume of tissue aspirated at P0 was assessed in adult ferrets by examination of Nissl-stained sections. In the six animals used for electrophysiological recording, we estimated that between 40 and $90 \%$ of the superficial layers had been removed from the right SC. In most cases, $\sim 50 \%$ of the superficial SC was left intact. The aspirated area extended forward from the caudal end of the SC and, in one animal, included part of the dorsal inferior colliculus. Auditory data from this animal were, therefore, not included in this study. In most cases, the neonatal surgery removed the stratum griseum superficiale (SGS) across the full mediolateral extent of the SC, although we never observed any damage to the contralateral SC. Occasionally, the extreme medial or lateral regions of the SGS were found to be intact. This is illustrated by

$\leftarrow$

dorsal views of the left SC on which the green (open area) and red (filled area) injection sites have been plotted. The separation of the green and red injection sites was systematically varied across animals; the distance between the centers of the injection sites in the SC is given below each dorsal view. The retinas were sampled at $500 \mu \mathrm{m}$ intervals, and the number of labeled cells was counted in a sample box of $125 \times 125 \mu \mathrm{m}$. The isodensity contours for cells containing green beads and cells containing red beads have been plotted separately ( fine hatching for green and bold dashes for red). For each retina and for each population of cells, the maximum number of cells in a sample box was noted, and the isodensity contours reflect $20 \%$ intervals of this peak density. Note that the separation between the retinal loci most heavily labeled by red or green beads varies with the spacing of the injections in the SC. $S$, Superior; $T$, temporal; $I$, inferior; $N$, nasal; $L$, lateral; $R$, rostral; $M$, medial, $C$, caudal. 

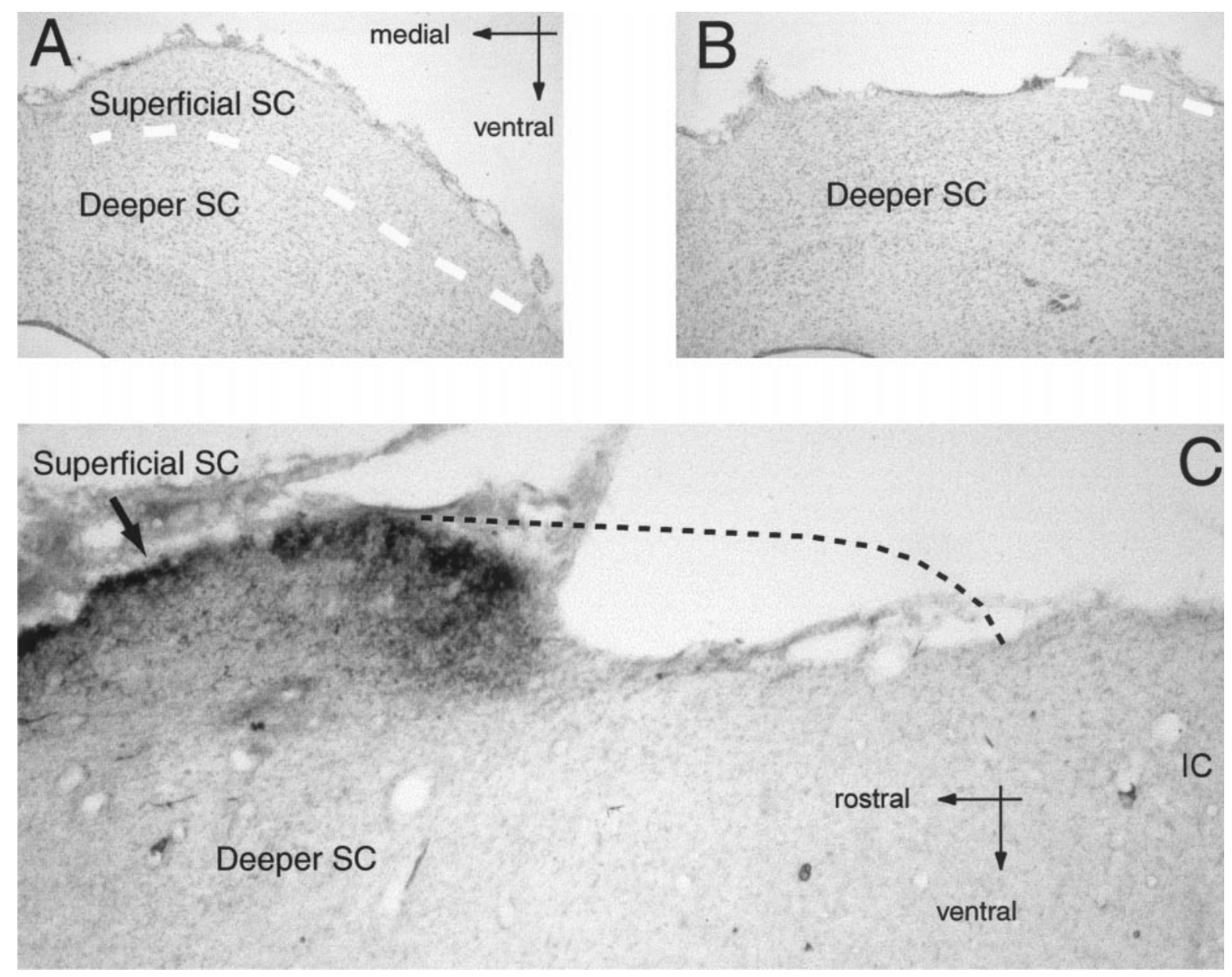

Figure 3. Partial lesions of the superficial layers of the SC. $A, B$, Nissl-stained coronal sections through two regions of the right SC of an adult ferret in which the caudal pole of the superficial layers had been removed on P0. In each case, the white dashed lines indicate the lower border of the stratum opticum. This border was determined by a careful examination of the sections using both light-field and dark-field illumination and by comparison with other sections of the ferret SC that were treated with a myelin stain. The rostral section $(A)$ shows intact superficial layers, whereas, in the caudal section $(B)$, these layers are missing across most of the mediolateral extent of the nucleus. $C$, Parasagittal, Nissl-stained section of the midbrain of an adult ferret in which the caudal region of the superficial layers had been aspirated at P0. Two days before the terminal recording experiment, this animal received an intraocular injection of $\left[{ }^{3} \mathrm{H}\right]$ proline. The midbrain was then prepared for autoradiography. The terminal labeling in the remaining portion of the superficial layers of the SC is shown by the distribution of black silver grains. At this mediolateral level of the SC, the superficial layers would normally extend more caudally as indicated by the black dashed line. In this animal, $\sim 50 \%$ of the superficial region was removed by the neonatal surgery. $S C$, Superior colliculus; $I C$, inferior colliculus.

the coronal sections shown in Figure $3, A$ and $B$, which are taken from one of the animals used for recording. The section in Figure $3 A$ shows a rostral region of the $\mathrm{SC}$ where the superficial layers are completely intact, whereas most of the SGS and stratum opticum (SO) are missing from the more caudal section in Figure $3 B$. As an additional means of estimating the extent of the neonatal aspiration, we prepared parasagittal sections from three of the ferrets for autoradiography after injecting $\left[{ }^{3} \mathrm{H}\right]$ proline into the contralateral eye $2 \mathrm{~d}$ before the recording experiment. An example is shown in Figure $3 C$, which illustrates that the labeling of retinocollicular terminals is restricted to the remaining rostral portion of the superficial layers.

At least part of the SO appeared to be intact in many sections. Occasional sections from some, but not all, animals showed evidence of localized damage to the dorsal part of the stratum griseum intermediale (SGI). Because of the extensive intrinsic connections found within the deeper layers of the SC (Behan and Kime, 1996; Meredith and Ramoa, 1998), the data recorded in regions of the SC where any of the SGI appeared to be missing were excluded.
In each of the lesioned animals, we mapped the visual receptive fields of multiunit activity recorded in the remaining, intact superficial layers. The variation in visual best azimuth with recording site for these electrode penetrations is shown in Figure $4 A$. Multiunit data obtained from the left, control SC in the same animals are shown in Figure $4 B$. The rostrocaudal extent of the remaining superficial layers of the $\mathrm{SC}$ on the lesioned side was sampled at intervals of $200 \mu \mathrm{m}$ or less, whereas larger electrode track separations were used for other parts of the nucleus. Histological reconstructions of the recording sites confirmed that the absence of data points in the caudal part of Figure $4 A$ closely matched the extent of the superficial layer lesion. Consequently, ferret 9454, which had the smallest lesion, yielded the most data points.

The visual best azimuths of the multiunit responses recorded in the remaining superficial layers tended to lie within or close to the range of values represented in this part of the nucleus in normal, adult ferrets, although most of the data from ferrets 9349 and 9456 fell just outside the normal range (Fig. $4 A$ ). The region of the visual field mapped in each animal was determined by the size 


\section{A Operated side: visual map in remaining part of superficial layers}

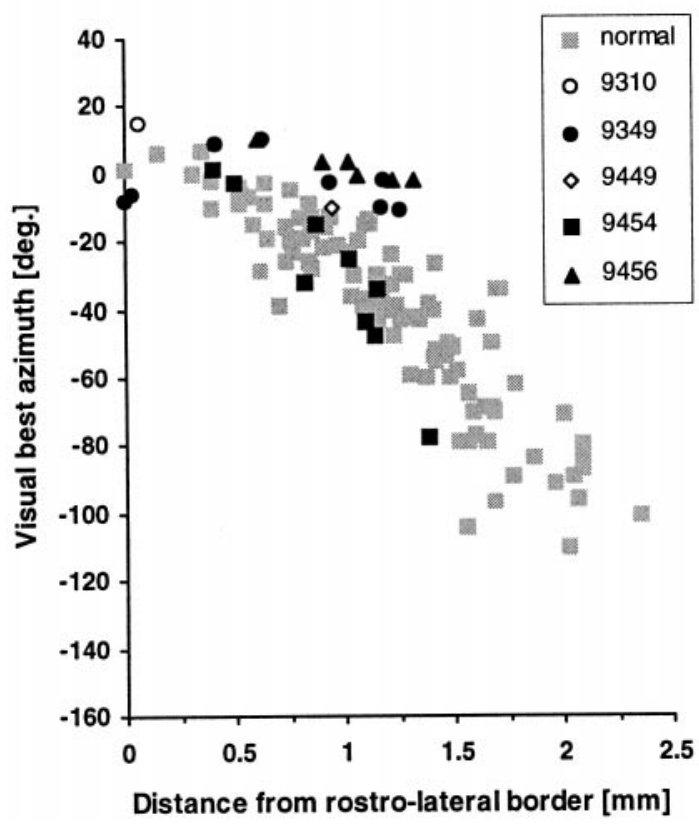

B Control side: visual map in superficial layers

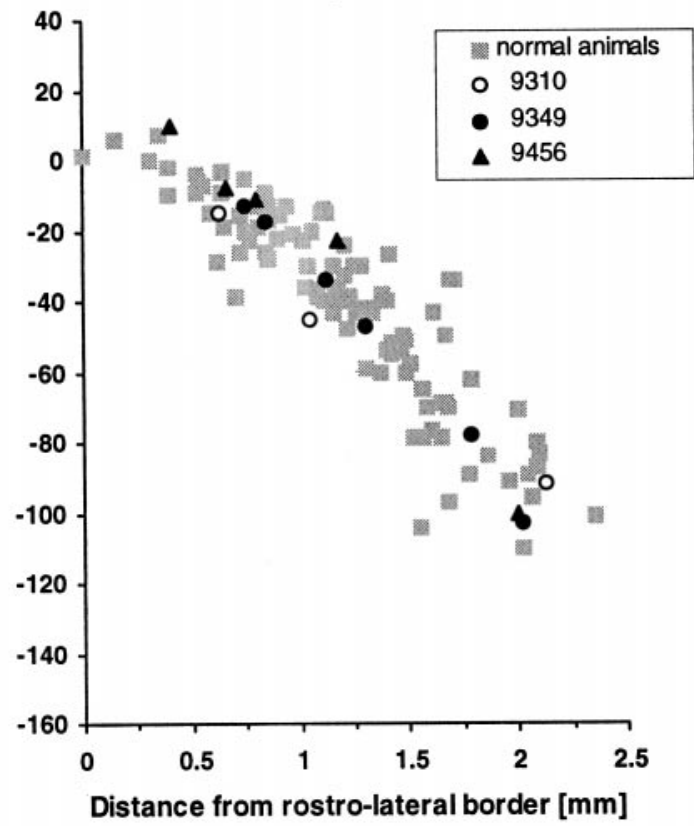

Figure 4. Effect of neonatal aspiration of the caudal part of the superficial layers of the right SC on the visual representation. A, Filled or open symbols indicate the multiunit visual best azimuths recorded in the remaining rostral portion of the superficial layers of the right SC. Different symbols are used for different animals. Note that the volume of the superficial layer region that was left intact and, therefore, the number of electrode penetrations that could be made varied between the animals. B, Filled or open symbols indicate the multiunit best azimuths plotted against the full extent of the superficial layers of the left, unoperated SC. In both panels, the background gray symbols show the distribution of multiunit visual best azimuths along the rostrocaudal axis of the SC in normal adult ferrets (King and Carlile, 1993; Schnupp et al., 1995).

of the lesion and, therefore, did not include the posterior locations that are normally represented in caudal SC. In contrast, the visual representation on the control side (Fig. 4B) was entirely normal.

For most recording sites in the residual superficial layers of the right $\mathrm{SC}$, we also isolated single units and mapped their receptive fields. Differences between single and multiunit visual receptive fields have been reported after neonatal, partial SC lesions in hamsters (Finlay et al., 1979; Pallas and Finlay, 1989) and visual deprivation in Xenopus (Keating and Kennard, 1987). We therefore examined the relationship between the best azimuths of the single-unit responses and their rostrocaudal location in the superficial layers (Fig. 5). Many of the single-unit best azimuths fell outside the range covered by the multiunit data from normal adult ferrets, typically yielding more nasal best azimuths than would have been expected from their recording sites. Most of these data were recorded from ferrets 9349 and 9456, which also gave more nasal multiunit receptive fields than in normal animals. This was not caused by abnormal eye position in these animals; the azimuthal coordinates of the back-projected optic disc position were, in both cases, within $1^{\circ}$ of the mean value based on 38 normal, adult ferrets. The apparent anterior shift in the distribution of visual best azimuths may reflect a change in the curvature of part of the SC after the neonatal superficial layer lesion. Alternatively, it is possible that there may be an expansion in the representation of the central visual field in these two animals.

In none of the ferrets did we observe any indication of a compression of the visual field representation analogous to that reported for the hamster SC after partial lesions of the superficial layers at birth (Finlay et al., 1979; Pallas and Finlay, 1991). We confirmed anatomically that neonatal lesions do not appear to

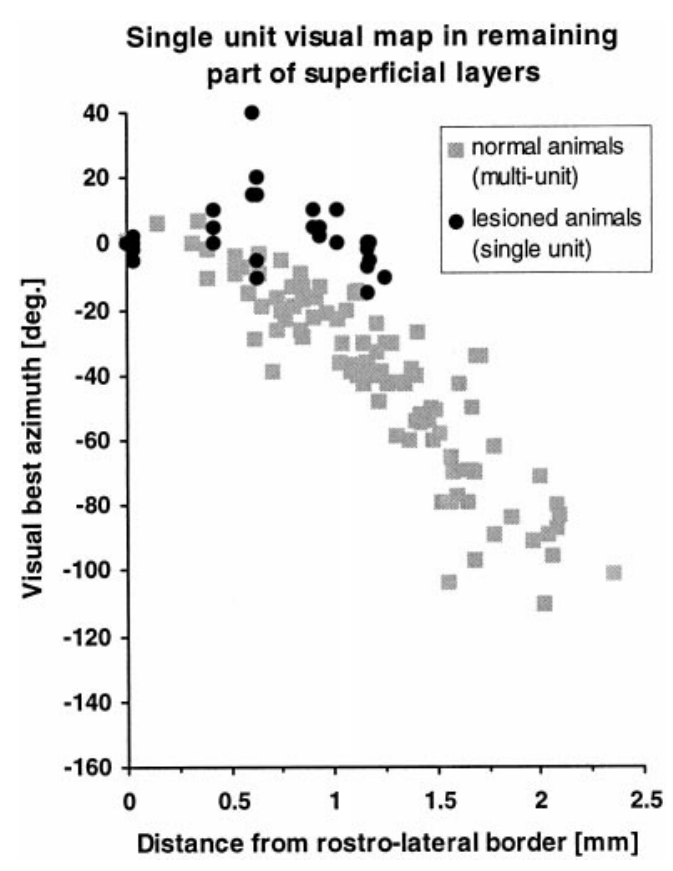

Figure 5. Single-unit visual best azimuths (filled symbols) recorded in the remaining rostral part of the superficial layers of the right SC in adult ferrets that had received partial superficial layer lesions at P0. Because of insufficient single-unit recordings from control animals, these data are superimposed on the distribution of multiunit visual best azimuths obtained from normal, adult ferrets ( gray symbols). 


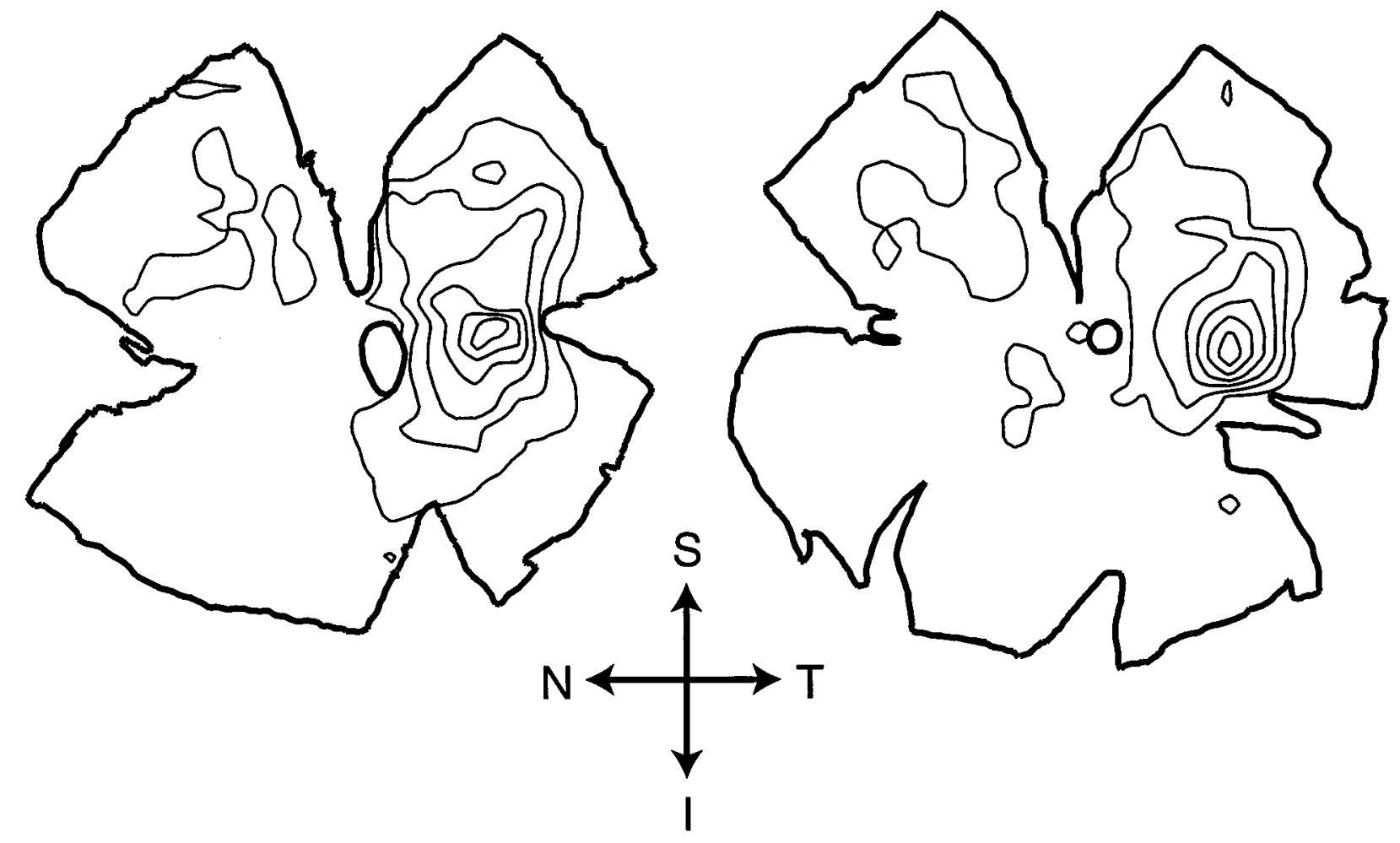

Figure 6. Flattened whole mounts of the retina in two adult ferrets showing the distribution of retrogradely labeled ganglion cells after multiple injections of HRP into the contralateral SC. The caudal part of the superficial layers of the SC had been aspirated at P0. The retinas were sampled at $500 \mu \mathrm{m}$ intervals, and the number of labeled cells was counted in a sample box of $125 \times 125 \mu \mathrm{m}$. For each retina, the maximum number of cells in a sample box was noted, and the isodensity contours reflect $10 \%$ intervals, beginning at $10 \%$ of this peak density. The smallest contours, near the peak, are not shown. Note that the labeling is concentrated in the temporal retina. The two retinas differ in the extent to which inferior retina is labeled, which reflects a difference in the medial spread of the HRP injections in the SC. $S$, Superior; $T$, temporal; $I$, inferior; $N$, nasal.

induce a reorganization of the visual representation in the residual superficial layers by making multiple HRP injections into the SC. Figure 6 shows the distributions of retinal ganglion cells, retrogradely labeled from the SC, in two adult ferrets in which a caudal ablation of the superficial layers of the SC had been made on the day of birth. The isodensity contours again indicate the relative density of labeling compared with the peak local density in each retina. The outer contour line represents $10 \%$ of the peak density. Heavy labeling was found in the retina temporal, but not nasal, to the optic disc; no region within the nasal part of the retina reached $20 \%$ of the peak density. In particular, there was no evidence of a horizontal streak extending into nasal retina. In normal animals, the streak extends nasally, such that the density of the retinocollicular projection in midnasal retina is $\sim 75 \%$ of that found at the area centralis (FitzGibbon et al., 1996). Although at a low density, a number of ganglion cells were labeled in the nasal retina of the ferrets that had received neonatal caudal $\mathrm{SC}$ lesions. Whether these reflect rescue of the relatively small numbers of early topographic errors or a rewiring of cells that originally projected to caudal SC is not clear.

\section{Effect of partial, neonatal lesions of the superficial layers on the responses of neurons in the deeper layers of the superior colliculus}

Distribution of acoustically responsive units

We were able to record acoustically responsive units throughout the intermediate and deep layers of the SC, both under the lesioned and nonlesioned areas. Auditory units were encountered immediately ventral to the lesion in the upper tier of the SGI where this formed the new dorsal surface of the nucleus. Azimuth response profiles were obtained for 126 units on the lesioned side and for 41 units on the control side. Of the units recorded on the lesioned side, 31 were found in electrode tracks that passed vertically through the remaining superficial layers from which visual responses were mapped. For brevity, the data obtained from these penetrations will be referred to as the $\mathrm{S}+$ condition. The other 95 auditory units were recorded in the part of the SC where the superficial layers were missing, as indicated by a lack of characteristic visual activity and confirmed by subsequent histological reconstruction of the electrode penetrations. These units were recorded in three animals, with the majority coming from ferrets 9310 and 9349. They are referred to in the following sections as the $\mathrm{S}-$ condition, because they were unlikely to have received direct superficial layer inputs during postnatal development.

\section{Bimodal units in the intermediate and deep layers}

Once the auditory azimuth profile had been mapped, we tested some of the auditory units for visual sensitivity by positioning a bright flashing LED either at the same angle from which the strongest auditory response had been evoked or directly in front of the eye so that it filled most of the visual field. Of the 14 units in the $\mathrm{S}+$ condition tested in this way, five $(36 \%)$ were shown to be bimodal. In the $\mathrm{S}-$ condition, 35 of 58 auditory units tested $(60 \%)$ also responded to visual stimulation, even though the superficial visual layers had been removed. Most of these bimodal units were found in the SGI. Only four units were tested in this 
way on the control side. Of these, two were found to be bimodal. The proportion of bimodal cells found in the $\mathrm{S}-$ condition is similar to that reported for the normal cat SC (Meredith and Stein 1986, 1990).

\section{Auditory sensitivity and responsiveness}

The response strengths at the best loudspeaker position varied from $0.3-7.0$ evoked spikes per presentation. The mean response at the best speaker position was highest in the $\mathrm{S}-$ condition with 1.46 evoked spikes per presentation $( \pm 1.17 \mathrm{SD})$, compared with $1.32( \pm 1.04)$ in the $S+$ condition and $0.90( \pm 0.66)$ on the unoperated control side. The difference between the $\mathrm{S}-$ condition and the unoperated side is statistically significant (Wilcoxon rank sum test, $p=0.01$ ). Unit thresholds also appeared to be significantly lower on average in the $\mathrm{S}-$ condition (mean $16 \mathrm{~dB}$ SPL) than in the $\mathrm{S}+$ condition $(25 \mathrm{~dB}$ SPL, Wilcoxon rank sum tests, $p=0.01$ ) or on the control side ( $22 \mathrm{~dB}$ SPL, $p=0.004$ ). Lower average thresholds for the $\mathrm{S}-$ units were observed consistently in every animal. The thresholds for the $\mathrm{S}+$ condition and the control side were not significantly different $(p=0.33)$.

\section{Auditory spatial tuning}

Because the superficial layer lesions were made along the rostrocaudal axis of the SC and, therefore, affected the nasotemporal extent of the visual field representation, we have restricted our analysis of auditory spatial tuning to the azimuthal dimension. As in previous studies (Schnupp et al., 1995), we assigned the azimuth response profiles to different categories and compared the relative proportions in each with those previously reported for normal, adult ferrets at comparable sound levels with respect to unit threshold. On the control side, $70 \%$ ( \pm SE of $7 \%$ ) were classified as tuned, compared with $71( \pm 8 \%)$ in the $\mathrm{S}+$ group, 79 $( \pm 4 \%)$ in the $S-$ group, and $77( \pm 5 \%)$ in the normal, adult population. Using a statistical test for comparing percentages (Bailey, 1981), we found that the proportion of tuned units in the four groups did not differ significantly from one another. The response profiles for the majority of the remaining units were classed as either bilobed or broad. More units exhibited ambiguously tuned bilobed response profiles in the $\mathrm{S}-$ group than in any of the other groups and were found in roughly equal proportion in ferrets 9310 and 9349. The difference between the proportion of bilobed response profiles in the $\mathrm{S}-$ group (17 of 95 units) and in the normal, adult ferrets ( 3 of 52 units) just reached significance (one-tailed test, $p<0.05$ ).

To assess the sharpness of auditory spatial tuning, we measured the width of each azimuth response profile in which the response had fallen to $50 \%$ of maximum (50\% bandwidth). As in previous studies, we included in this estimate all regions of the response profile that exceeded the $50 \%$ level, whether or not these regions were classified as separate peaks. The distribution of $50 \%$ bandwidths is shown by the histograms in Figure 7 . The spread in the histograms in each of the three experimental conditions is very close to that found in normal, adult ferrets. As expected from the very similar proportions of tuned units, we found no differences in the mean $50 \%$ bandwidths between any of the groups $\left(105^{\circ}\right.$ for normal, adult ferrets, $114^{\circ}$ on the control side, $104^{\circ}$ in the $\mathrm{S}+$ group, and $112^{\circ}$ in the $\mathrm{S}-$ group; Wilcoxon rank sum tests, $p>$ $0.25)$.

\section{Auditory topography}

The topographic order in the auditory representation was assessed by plotting the azimuthal best position (where the maxi-
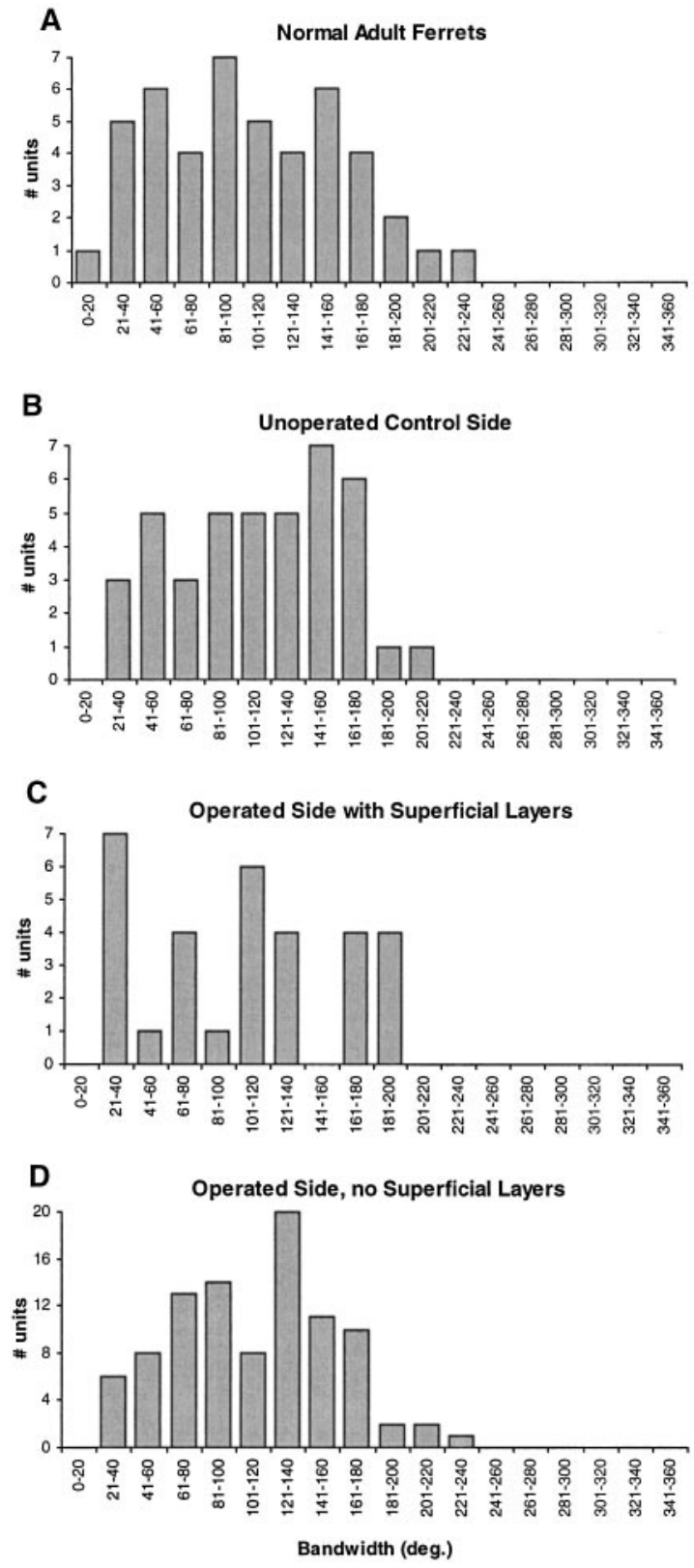

Figure 7. Distribution of 50\% azimuth profile bandwidths (width of each azimuth response profile in which the response had fallen to $50 \%$ of maximum) in normal, adult animals and in adult ferrets that had received a partial lesion of the superficial layer lesions on $\mathrm{P} 0$. The data from normal animals are from King and Carlile (1993).

mum response was obtained) against the rostrocaudal distance of each unit from the rostrolateral border of the SC and, when possible, against the visual best azimuth of multiunit activity recorded in the superficial layers of the same electrode penetration. Figure 8 illustrates how the auditory best azimuths vary with recording site in the $\mathrm{S}-$ and $\mathrm{S}+$ groups from the right $\mathrm{SC}$ and in the left, unoperated SC. In each case, the data are superimposed on data obtained in previous studies from normal, adult ferrets. Only tuned units, which represented the great majority of the auditory responses recorded in each group, are included in this and subsequent figures. This is partly for reasons of clarity and partly because the proportion of tuned units did not differ significantly across the groups. 
Auditory representation where superficial layers are missing

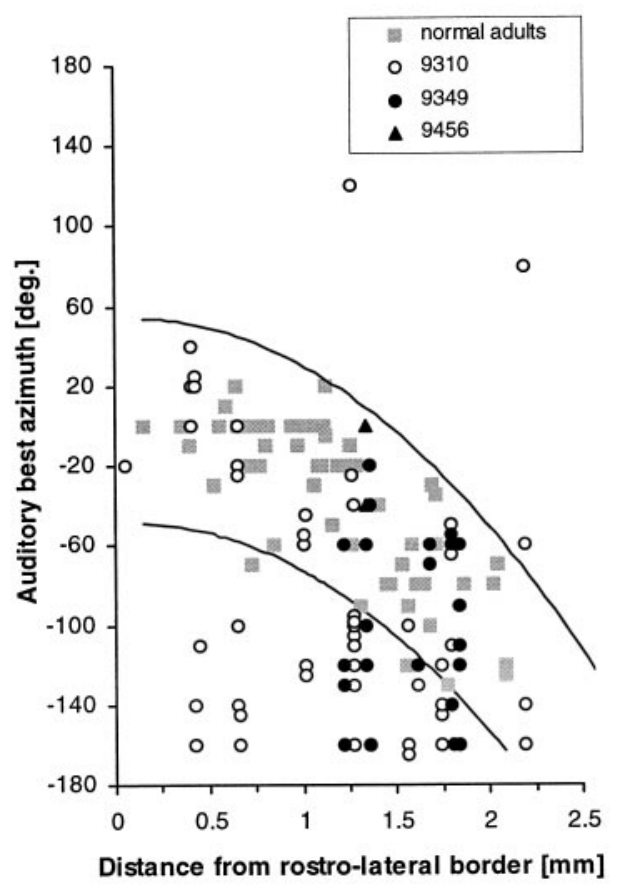

Auditory representation under remaining superficial layers

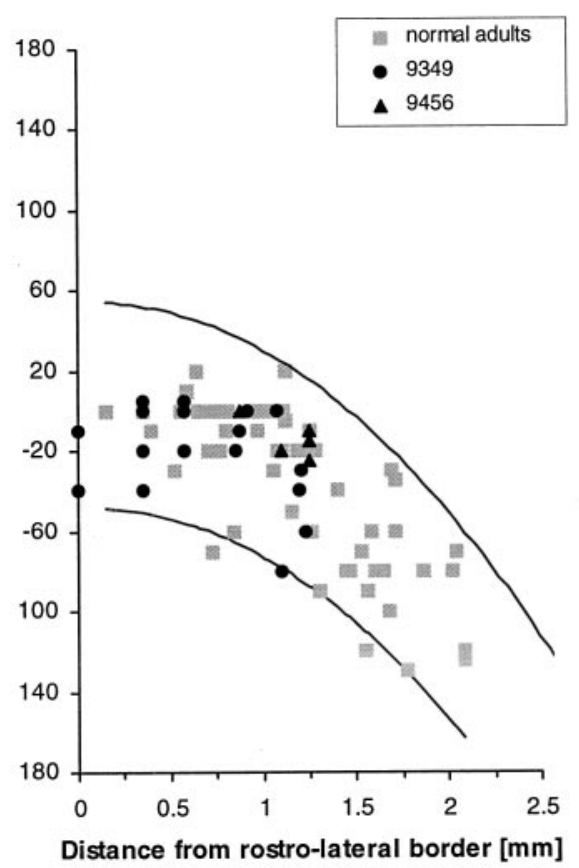

Auditory representation on unoperated (control) side

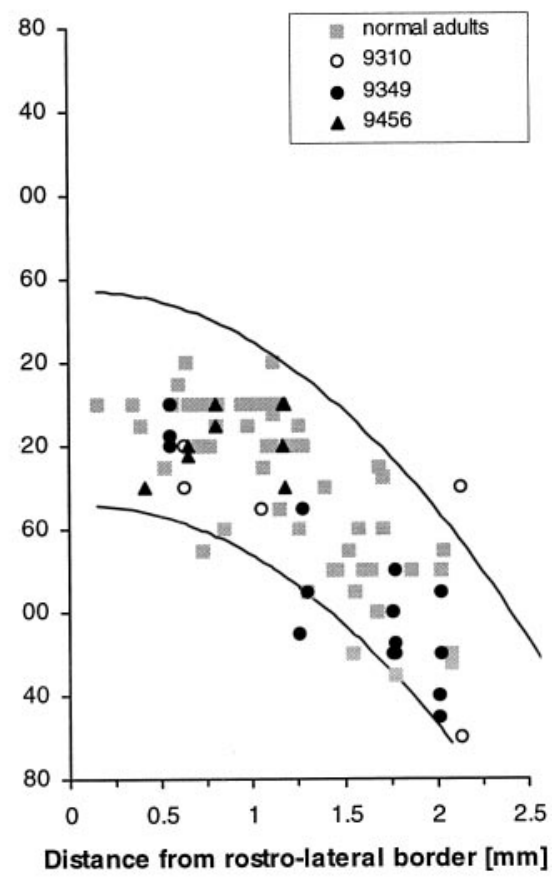

Figure 8. The effect of neonatal superficial layer lesions on auditory azimuth topography. The filled and open symbols show the auditory single-unit best azimuths plotted against the rostrocaudal location of their recording sites in adult ferrets that had received partial lesions of the superficial layers of the right SC on P0. The different symbols represent data from different animals. The gray symbols indicate data obtained from normal adult controls during previous studies, and the lines represent $95 \%$ confidence intervals for those data. Left, Representation of sound azimuth in the deeper layers of the right SC ventral to the superficial layer lesion. Middle, Representation in rostral parts of the right SC where the superficial layers were intact. Note that the rostrocaudal range of recording sites overlaps in these two groups because of interanimal variations in the extent of the lesion. Right, Representation of sound azimuth on the unoperated, left side of the SC.

It is clear from Figure 8 that a large proportion of auditory units in the $\mathrm{S}$ - group (left panel) were tuned to sound directions that fell outside the normal range of values associated with each recording site. Although the majority were tuned to contralateral locations, a few best azimuths were also found well into the ipsilateral hemifield. In contrast, the auditory best azimuths of all the tuned units recorded beneath the intact superficial layers fell within the normal range (Fig. 8, middle panel), as did those recorded in the unoperated, left SC (Fig. 8, right panel). It therefore appears that a partial lesion of the superficial layers led to degradation in the order of the auditory representation that was localized to the region of the deep SC from which the superficial layers had been removed. The range of best azimuths represented in each region of the SC was very similar among all the animals that contributed to each group.

In Figure 9, different symbols have been used to differentiate between auditory units shown to be bimodal and those that either did not appear to respond to or were not tested with visual stimuli. Many of the units with aberrant auditory best positions were also visually responsive. This appears to indicate that, in the absence of directly overlying superficial layers, visual inputs from other sources do not rescue the auditory topography. We were able to hold the units for long enough to measure their visual receptive fields in only a few cases. For some of these bimodal units, we noted a close correspondence between the auditory and visual best azimuths, whereas, in others, these values varied by $40^{\circ}$ or more. We have too few data to be able to describe how the visual azimuths of units recorded in the deeper layers of these animals vary with their rostrocaudal recording site, or whether neonatal removal of the overlying superficial layers alters this relationship.

The auditory representation was quantified by comparing the observed best azimuth for each unit with the value predicted for that recording site from the normal adult map. King and Carlile (1993) have previously reported that the polynomial equation $Y=$ $15.9-13 X-24.2 X^{2}$ provides the best fit for the auditory azimuth map obtained at sound levels of 25-30 dB above unit threshold (where $X$ is the standardized electrode distance from the rostrolateral pole of the SC in millimeters, and $Y$ the auditory best azimuth in degrees). The distribution of auditory topography errors, which represent the difference between the measured best azimuths and the value predicted from this polynomial function, are shown for each of the groups by the histograms in Figure 10. The histograms were unimodal and centered on low error values. As expected from Figure 8, the width of the histograms for both the left SC control data (Fig. 10B) and the S+ group from the right SC (Fig. 10C) was very similar to that found for the normal, adult ferrets (Fig. 10A). In contrast, a much greater spread in topography errors was observed for the $\mathrm{S}-$ group (Fig. 10D).

The variances of these topography errors are given in Table 1, together with the $p$ values of the $F$ tests that were used to compare the variances. Because the scatter in the normal, adult data increases slightly toward the caudal end of the SC, we have restricted these comparisons to data obtained from the same rostrocaudal extent of the nucleus. In the $\mathrm{S}-$ condition, almost 


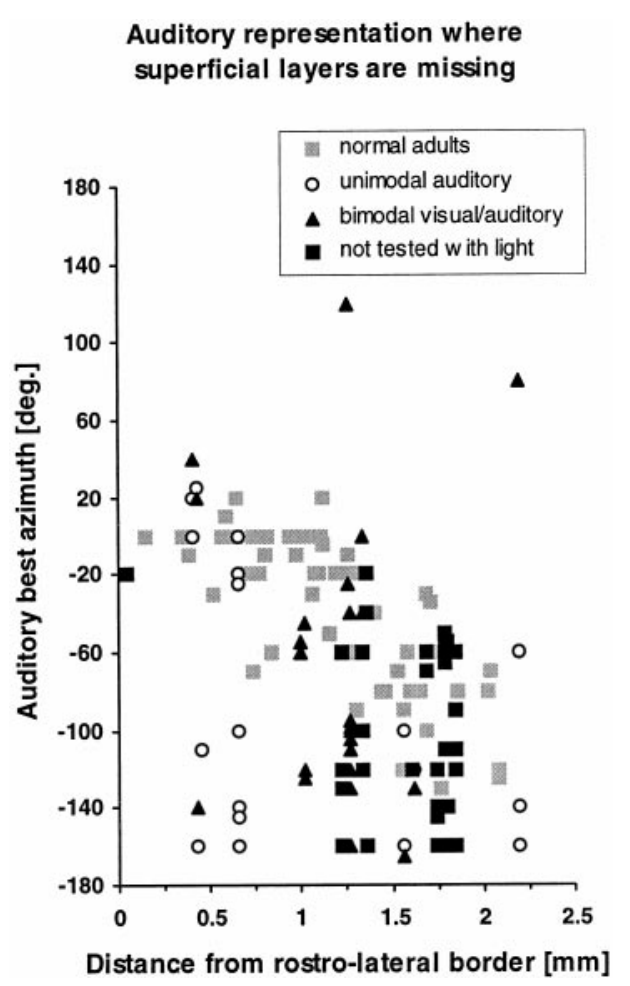

Figure 9. Representation of sound azimuth in the deeper layers of the right SC ventral to the superficial layer lesion. These data are replotted from Figure 8 . This time different symbols are used to indicate whether the auditory units were shown to be visually responsive.

the entire SC was sampled, given that in one of the ferrets only $\sim 10 \%$ of the superficial layers was left intact. The auditory units comprising the $\mathrm{S}+$ group were, however, restricted to the rostral $1.25 \mathrm{~mm}$ of the SC. The $\mathrm{S}+$ data were, therefore, compared with a subset of the normal adult data from an equivalent rostral region. The variance of the topography errors for the $S-$ group, where the superficial layers were missing, was significantly greater than the variance associated with the $\mathrm{S}+$ group, the left unoperated SC, or the normal control animals. On the other hand, there was no difference in the scatter in the auditory representation between the rostral region of the right SC with intact superficial layers and either of the two control groups.

Because of the possibility that auditory units located in the SGI might be more affected than those in the stratum griseum profundum (SGP) by aspiration of the superficial layers, we also measured the topography errors in the $\mathrm{S}-$ group at different depths below the new surface of the SC. However, we found no difference in the variance of the topography errors between auditory units located within $600 \mu \mathrm{m}$ of the surface and those found in deeper regions of the SC $(p=0.21)$.

\section{Auditory-visual alignment}

We also examined the relationship between the auditory best azimuths of deeper layer units and the visual best azimuths of multiunit activity recorded in the overlying superficial layers. A close correspondence was found between the restricted visual and auditory representations in the rostral SC for the $\mathrm{S}+$ group (Fig. 11, left panel). The auditory best positions of two units were somewhat more contralateral than their superficial layer visual counterparts. These units were, however, recorded from a region of the deeper layers that was beneath the edge of the lesion. On
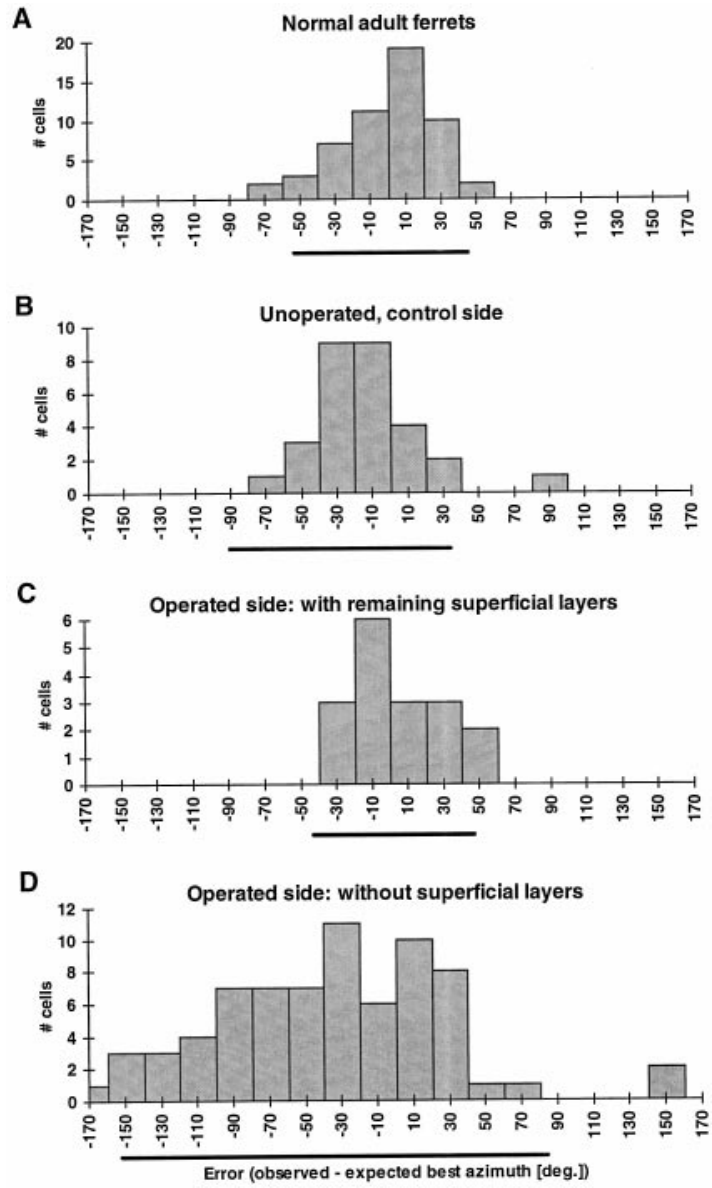

Figure 10. The effect of neonatal partial lesions of the superficial layers of the right SC on the topography of the auditory representation. The histograms plot the angular difference between the azimuthal best positions (where the maximum response was obtained) of tuned auditory units and the expected value at each recording site, which was derived from the polynomial function that produced the best fit for the map of sound azimuth in normal adult ferrets. The bar below each histogram is centered on the mean topography error and extends to two SDs on either side. The spread in the distribution of topography errors is clearly widest in panel $D$, which refers to the part of the SC where the superficial layers were missing.

the left control side, the correspondence between the auditory and visual best azimuths throughout the rostrocaudal extent of the SC closely resembled that seen in normal, adult ferrets (Fig. 11, right panel).

The difference between the auditory and visual coordinates of units recorded in the same vertical electrode penetrations is shown by the histograms in Figure 12. Like the auditory topography errors shown in Figure 10, the auditory-visual misalignments in the normal, adult group (Fig. 12A), on the left control side of the SC (Fig. 12B), and in the S+ condition on the right side (Fig. 12C) were small and approximately normally distributed. A series of $F$ tests did not reveal any statistical difference in the variance in the auditory-visual misalignments between any of the three groups (Table 2).

\section{Partial lesions of the superficial layers in adult ferrets}

The data presented so far indicate that partial lesions of the superficial layers in P0 ferrets result in a localized disruption of topographic order in the auditory representation in the deeper layers. This disrupted topography could represent a failure of the 
Table 1. Variance (scatter) in auditory topography errors

\begin{tabular}{|c|c|c|c|c|}
\hline \multirow[b]{2}{*}{ Condition } & \multirow[b]{2}{*}{$n$ (cells) } & \multirow[b]{2}{*}{ Variance } & \multicolumn{2}{|c|}{$p$ Value of Comparison with } \\
\hline & & & Normal & Unoperated control side \\
\hline Normal animals, full extent & 54 & 664 & & \\
\hline Control side, full extent & 29 & 940 & 0.272 & \\
\hline Neonatal SC lesion, no superficial layers & 75 & 3908 & $<<0.0001$ & $<<0.0001$ \\
\hline Normal animals, rostral part & 32 & 447 & & \\
\hline Control side, rostral part & 15 & 752 & 0.32 & \\
\hline \multirow[t]{2}{*}{ Neonatal SC lesion, with superficial layers } & 22 & 607 & 0.53 & \\
\hline & & & \multicolumn{2}{|c|}{ No superficial layers } \\
\hline Neonatal SC lesion, no superficial layers & 75 & 3908 & & \\
\hline Neonatal SC lesion, with superficial layers & 22 & 607 & $<<0.0001$ & \\
\hline
\end{tabular}

\section{Visual - auditory alignment in right SC, caudal superficial layers ablated}

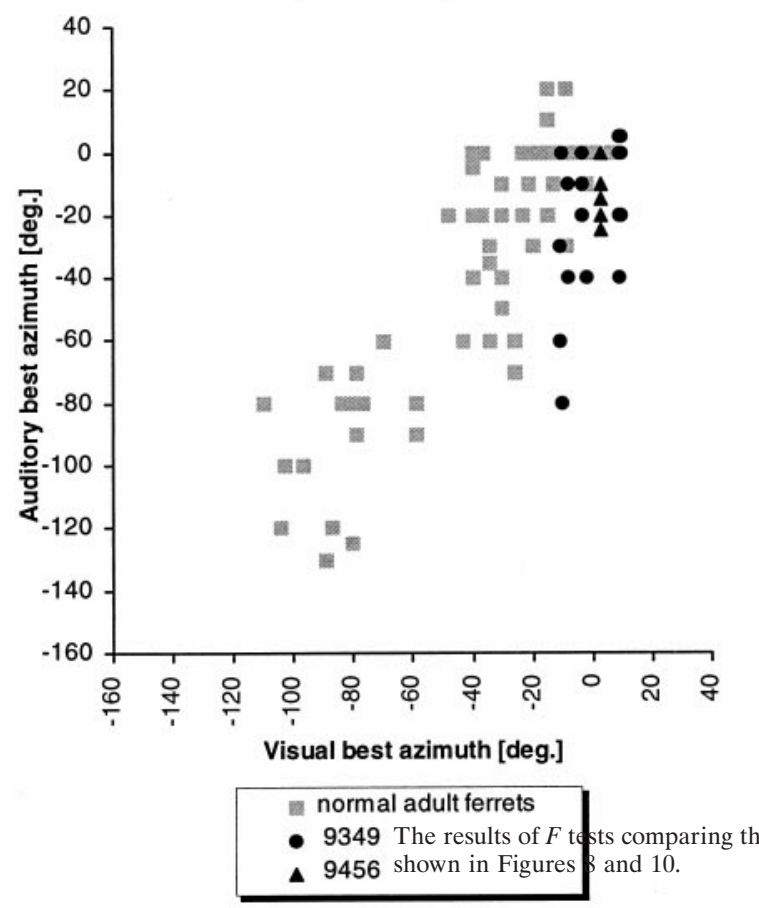

Visual - auditory alignment on left, unoperated control side

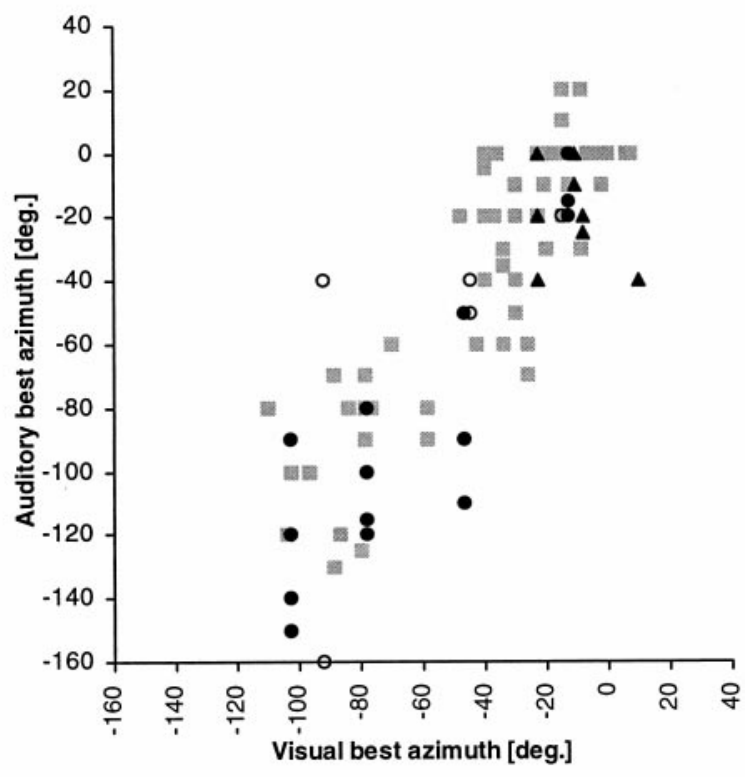

Figure 11. Relationship between the representations of visual azimuth in the superficial layers and auditory azimuth in the deeper layers of the superior colliculus. For each vertical electrode penetration, the best visual azimuth is plotted against the auditory best position. Left, Filled symbols indicate the responses recorded from the rostral region of the right superior colliculus, where the superficial layers were still intact after a lesion on P0. Right, Filled and open symbols indicate the data obtained from the left, unoperated side. In both panels, the gray symbols represent data from normal adult controls that were used in earlier studies.

auditory space map to develop normally or it might be a consequence of damage to local circuits within the SC that is unrelated to developmental superficial-deep layer interactions. To distinguish between these possibilities, we also examined the consequences of making partial lesions of the superficial layers in two adult ferrets. Recordings made $\sim 4$ months after the surgery revealed the presence of a normal representation of sound azimuth ventral to both lesioned and nonlesioned regions of the SC. This is shown in Figure 13, which shows the relationship between the auditory best azimuths of the tuned units and the rostrocaudal location of the recording electrode. Thirty-two auditory units (of a total of 38) were classified as tuned to single sound directions. Histological reconstruction of the electrode tracks revealed that 23 of these units were recorded in regions of the SC from which the superficial layers had been removed. Figure $13 A$ shows that the best azimuths for virtually all of these units fell within the normal range. Moreover, the variance in the auditory topography errors (Fig. 13B,C) did not differ significantly from that found in normal, adult controls (two-tailed $F$ test, $p=0.47$ ), indicating that, in contrast to the effects of neonatal lesions, the auditory representation beneath the adult lesion was unimpaired.

The lesions in the adult animals left small regions of the SC 
A

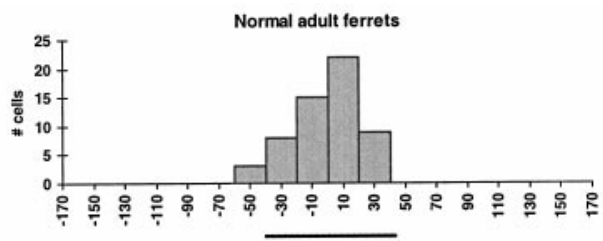

B

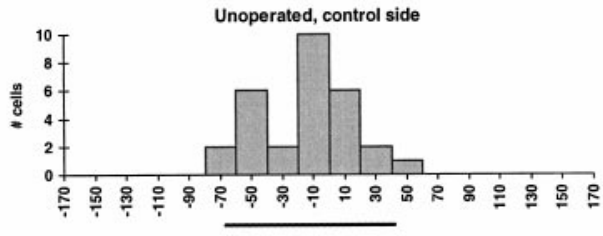

C

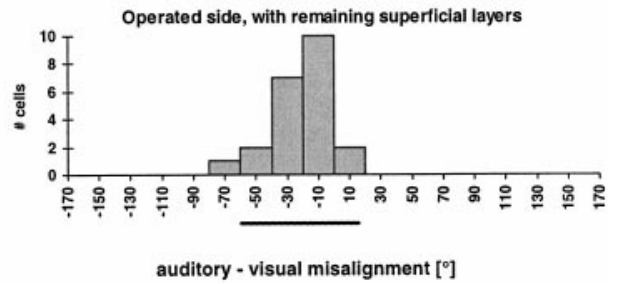

Figure 12. The effect of neonatal partial lesions of the superficial layers of the right SC on auditory-visual misalignments. The histograms plot the angular difference between the best azimuths of multiunit visual responses in the superficial layers and those of tuned auditory single units recorded in the deeper layers of the same electrode penetration. The bar below each histogram is centered on the mean misalignment and extends to 2 SDs on either side.

Table 2. Variance (scatter) in auditory-visual misalignments

\begin{tabular}{llll} 
& & & $\begin{array}{l}p \text { value of } \\
\text { comparison } \\
\text { with }\end{array}$ \\
\cline { 3 - 4 } Condition & $n$ (cells) & Variance & Normal \\
\hline Normal animals, full extent & 57 & 451 & \\
Unoperated side, full extent & 29 & 789 & 0.075 \\
Normal animals, rostral part & 32 & 416 & \\
Operated side, with superficial layers & 22 & 360 & 0.740
\end{tabular}

The results of $F$ tests comparing the variances in the auditory-visual misalignments in equivalent portions of the SC. Based on the data shown in Figures 11 and 12.

intact at both the rostral and caudal ends of the nucleus. Figure $13 A$ also includes the auditory best azimuths of nine units that were recorded in one of the two animals from the caudal pole of the SC where the superficial layers were intact. These units were tuned to very contralateral sound directions that closely matched the visual best azimuths of units recorded in this residual region of the superficial layers.

\section{DISCUSSION}

Representation of visual space in the superficial layers

Ablation of the caudal half of the optic tectum in adult goldfish (Gaze and Sharma, 1970) and frogs (Udin, 1977) produces a compression of regenerating retinotectal axons, so that the entire visual field becomes mapped over the remaining rostral region. A similar compression of the visual field representation can be induced by making partial SC lesions in neonatal hamsters (Finlay et al., 1979; Pallas and Finlay, 1989, 1991). In contrast, our physiological data indicate that partial removal of the superficial
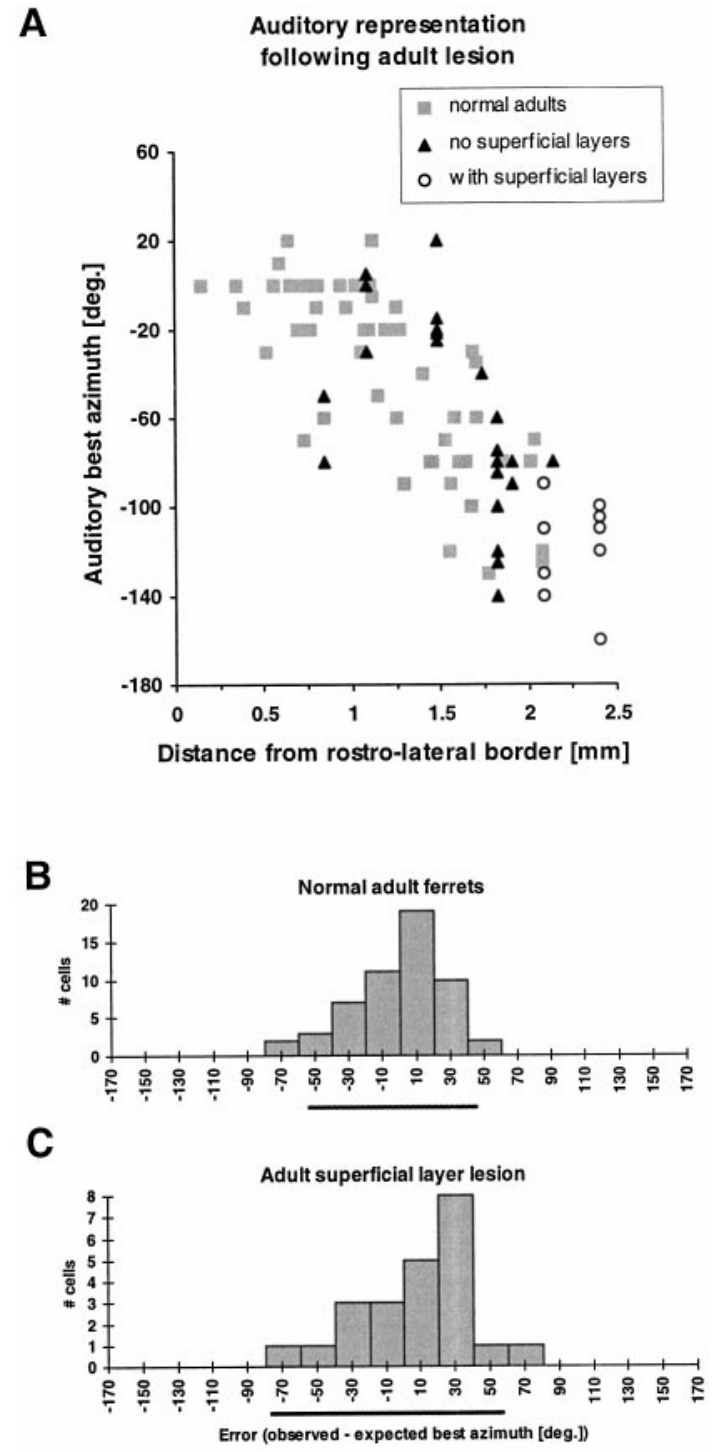

Figure 13. The effect of aspirating part of the superficial layers in adult ferrets on the topography of the auditory representation. $A$, Relationship between auditory best azimuth and rostrocaudal recording site. The filled triangles indicate the responses of units recorded in electrode tracks where the overlying superficial layers were missing. The open circles show data recorded in the extreme caudal part of the SC where the superficial layers were still intact. As in Figure 8, data from the normal adult control population are represented by the gray symbols. $B, C$, Distribution of auditory topography errors in the normal adult animals and in the ferrets that received partial superficial layer lesions in adulthood, shown as in Figure 10.

layers in P0 ferrets does not lead to a reorganization of the visual representation in the remaining region.

The failure to obtain map compression in the ferret SC probably does not reflect excessive target ablation (Pallas and Finlay, 1989). Although only $10 \%$ of the superficial layers was spared in one ferret, $50 \%$ or more remained in three of the others. The overall developmental state of the retinal projections at birth in the two species is also similar. Segregation of the uncrossed and crossed retinal axons has not occurred (Linden et al., 1981; Thompson et al., 1995), and the bulk of ganglion cell death has yet to happen (Tay et al., 1986; Henderson et al., 1988). What is different is the state of retinotopic order in the SC of newborn 
hamsters and ferrets. The pattern of retrograde labeling observed after SC injections of fluorescent microspheres indicates that the ferret retincollicular projection possesses a high degree of topographic order on $\mathrm{P} 0$. This is consistent with a recent anterograde tracing study by Chalupa and Snider (1998), which found that retinal axons in neonatal and fetal ferrets exhibit few targeting errors within the contralateral SC. In contrast, the retinocollicular projection in neonatal rats (Simon and O'Leary, 1992) and hamsters (Thompson and Cordery, 1994) is initially much more diffuse and depends on activity in the target tissue for its subsequent maturation (Simon et al., 1992).

\section{Auditory responses after superficial layer lesions}

The auditory responses were normal beneath the intact superficial layers, and their preferred stimulus directions closely matched those of the overlying visual units. In contrast, the range of auditory best azimuths corresponding to each recording site in the region of the SC below the superficial layer lesion was significantly greater than normal. This was true irrespective of the size of the lesion. It is unlikely that the neonatal surgery damaged the auditory afferents to the deeper layers of the SC. In ferrets, the ipsilateral BIN and ICX provide the principal auditory inputs, and the projection from the BIN is spatially ordered along the rostrocaudal axis of the SC (King et al., 1998). Anterograde tracing studies in mice (Wallace and Fredens, 1989) and cats (Kudo et al., 1984) have shown that afferent fibers from the BIN spread throughout the deeper layers of the SC but are concentrated primarily in SGI and in SGP. After tracer injections in the ferret BIN, the heaviest axonal labeling is found in SGP and, to a lesser extent, in the mid and upper regions of SGI (J. Baron, T. P. Doubell, and A. J. King, unpublished observations). As reported in the other studies (Kudo et al., 1984; Wallace and Fredens, 1989) some axons also extend into the lower part of SO. Nevertheless, the majority of these afferents course through regions of the SC that are well away from the lesioned area. Moreover, acoustically responsive neurons were found throughout the intermediate and deep layers, and their firing rates and thresholds were clearly unimpaired by the lesions. In addition, the azimuth bandwidths and the proportion of tuned units were no different from those recorded in the unoperated, left SC.

If the superficial layers transmit visual signals that contribute to the refinement of the developing auditory space map, the consequences of superficial layer lesions should mimic the effects on the auditory responses of visual deprivation. Disrupting the pattern of visual input during development by binocular eyelid suture leads to the emergence of an auditory representation that is abnormal in various ways (Knudsen et al., 1991; Withington, 1992; King and Carlile, 1993). In ferrets, both eyelid suture (King and Carlile, 1993) and superficial layer lesions degrade the precision of the auditory representation without affecting the azimuth-tuning bandwidths of individual units. In each case, many units are tuned to sound directions outside the normal range of values and the proportion of units with spatially ambiguous bilobed response profiles increases.

Auditory responses also develop abnormally in the SC of ferrets in which NMDA receptor antagonists are applied locally, via Elvax implants, during the period over which the auditory map normally matures. This results in more ambiguously tuned auditory units and greater scatter in the auditory representation (Schnupp et al., 1995). Both changes closely resemble the effects of superficial layer lesions, although, in contrast to the animals raised with Elvax implants, we did not observe a significant reduction in the proportion of tuned auditory units in the present study. The visual map in the superficial layers of the ferret SC was found to be unaffected after removal of the Elvax implants (Schnupp et al., 1995). It is, therefore, possible that NMDA receptor blockade disrupts the transmission of signals between the superficial and deeper layers, particularly in infancy when excitatory synaptic currents in the SC are dominated by these receptors (Hestrin, 1992; Wu et al., 1996; Shi et al., 1997).

Partial ablation of the superficial layers of the SC produces a localized disruption of the auditory representation if this procedure is performed at $\mathrm{P} 0$, but not in adult animals. Again, this is comparable to the effects of Elvax implantation because chronic application of NMDA receptor antagonists in adult ferrets does not affect the auditory map. The auditory representation is also most susceptible to altered visual cues during an early stage of development (Withington-Wray et al., 1990; King, 1993; Brainard and Knudsen, 1998).

\section{Do visual signals from the superficial layers guide the development of the auditory space map?}

A fairly well ordered map of visual space is found in the deeper layers of the cat SC, which shares the same axes as the superficial layer visual map (Meredith and Stein, 1990) and the deep layer auditory map (Middlebrooks and Knudsen, 1984). Many deep layer visual neurons also respond to sound (Gordon, 1973; Middlebrooks and Knudsen, 1984; Meredith and Stein, 1986, 1990; Schnupp et al., 1997), although, in cats, the responses to visual stimuli first appear $>1$ week after the first auditory responses (Wallace and Stein, 1997). The receptive fields of both modalities are progressively sharpened as the neurons begin to integrate multisensory signals. As long as the deeper layer visual responses possess some spatial tuning and topographic order, they could provide the signals that calibrate the developing auditory space map. An alternative template could be provided by the superficial layer neurons, which exhibit much smaller receptive fields, a more precise topography, and more reliable responses than their deeper layer counterparts (Meredith and Stein, 1990). Moreover, an adult-like visual map can be demonstrated in the superficial layers in cats (Kao et al., 1994), ferrets (King and Carlile, 1995), and primates (Wallace et al., 1997) as soon as the eyes open.

In the region of the SC below the superficial layer lesion, we found that most auditory neurons tested were also visually responsive. It seems likely that, as in cats (Hardy and Stein, 1988; Harting et al., 1992; Niida et al., 1997), these visual responses arise from extrastriate cortical areas rather than the superficial layers, as appears to be the case in hamsters (Mooney et al., 1992). Alternatively, they may reflect aberrant inputs from cortical or subcortical regions that normally innervate the superficial layers. In the cat, axons from the posteromedial lateral suprasylvian cortex enter the ipsilateral SC via the SO and arborize in the SGS, SO, and SGI (Bruce, 1993). We cannot say whether the superficial layer lesion altered the visuotopic organization in the deeper layers and are therefore unable to rule out the possibility that some of the descending corticotectal axons may have been damaged by this procedure. Nevertheless, the presence of visual activity below the lesion was clearly not sufficient to calibrate the spatial tuning of the auditory neurons. Consequently, it seems more likely that neurons within the superficial layers provide signals that actively guide the maturation of the underlying auditory space map.

Topographically organized connections between the superficial 
and deeper layers of the SC provide a means by which highly localized visual signals could influence the activity of acoustically responsive neurons. Our observation that auditory units recorded below the superficial layer lesion had significantly lower thresholds and higher mean responses than in other parts of the SC is consistent with a descending inhibitory influence from those layers. However, it has recently been demonstrated in SC slices from young tree shrews that excitatory postsynaptic currents can be generated in SGI neurons by electrical stimulation of the superficial layers (Lee et al., 1997). The largest currents resulted from activation sites dorsal to the intermediate layer neuron. Although action potentials were also recorded in these neurons, subthreshold excitatory currents could, in principle, provide sufficient depolarization to enhance simultaneous auditory activity in a location-specific manner and subsequently strengthen the synapses associated with those inputs.

Our findings would suggest that developmental interactions between visual and auditory inputs take place in the SC. However, in owls, prism rearing alters ITD tuning in both the SC and the ICX (Brainard and Knudsen, 1993). It is possible that the map of sound azimuth in the mammalian BIN, which provides the principal, spatially ordered auditory input to the SC (King et al., 1998), may also be influenced by altered visual experience, given that the ferret BIN contains bimodal neurons (Schnupp and King, 1997) and receives a spatially ordered projection from both the superficial and deeper layers of the SC (Baron et al., 1998).

\section{REFERENCES}

Annetts CSJ, Howse BPA, Hutchings ME, King AJ (1987) A digitally controlled vertical hoop system for the presentation of free-field auditory and visual stimuli. J Physiol (Lond) 382:21P.

Bailey NJT (1981) Statistical Methods in Biology. Cambridge UP.

Baron J, Doubell TP, King AJ (1998) Back projection from the superficial layers of the ferret superior colliculus to its primary source of auditory input in the inferior colliculus. Soc Neurosci Abstr 24: 1882.

Behan M, Appell PP (1992) Intrinsic circuitry in the cat superior colliculus: Projections from the superficial layers. J Comp Neurol 315:230-243.

Behan M, Kime NM (1996) Intrinsic circuitry in the deep layers of the cat superior colliculus. Vis Neurosci 13:1031-1042.

Brainard MS, Knudsen EI (1993) Experience-dependent plasticity in the inferior colliculus: a site for visual calibration of the neural representation of auditory space in the barn owl. J Neurosci 13:4589-4608.

Brainard MS, Knudsen EI (1998) Sensitive periods for visual calibration of the auditory space map in the barn owl optic tectum. J Neurosci 18:3929-3942.

Bruce LL (1993) Postnatal development and specification of the cat's visual cortioctectal projection: efferents from the posteromedial lateral suprasylvian area. Dev Brain Res 73:47-61.

Carlile S (1990) The auditory periphery of the ferret. I: Directional response properties and the pattern of interaural level differences. J Acoust Soc Am 88:2180-2195.

Carlile S, King AJ (1994) Monaural and binaural spectrum level cues in the ferret: acoustics and the neural representation of auditory space. J Neurophysiol 71:785-801.

Chalupa LM, Snider CJ (1998) Topographic specificity in the retinocollicular projection of the developing ferret: an anterograde tracing study. J Comp Neurol 392:35-47.

Finlay BL, Schneps SE, Schneider GE (1979) Orderly compression of the retinotectal projection following partial tectal ablation in the newborn hamster. Nature 280:153-155.

FitzGibbon T, Wingate RJ, Thompson ID (1996) Soma and axon diameter distributions and central projections of ferret retinal ganglion cells. Vis Neurosci 13:773-786.

Gaze RM, Sharma SC (1970) Axial differences in the reinnervation of the goldfish tectum by regenerating optic fibers. Exp Brain Res 10:171-181.

Gordon BG (1973) Receptive fields in deep layers of cat superior colliculus. J Neurophysiol 36:157-178.
Grantyn R, Ludwig R, Eberhardt W (1984) Neurons of the superficial tectal gray. An intracellular HRP-study on the kitten superior colliculus in vitro. Exp Brain Res 55:172-176.

Hall WC, Lee P (1997) Interlaminar connections of the superior colliculus in the tree shrew. III: The optic layer. Vis Neurosci 14:647-661.

Hardy SC, Stein BE (1988) Small lateral suprasylvian cortex lesions produce visual neglect and decreased visual activity in the superior colliculus. J Comp Neurol 273:527-542.

Harting JK, Updyke BV, van Lieshout DP (1992) Corticotectal projections in the cat: anterograde transport studies of twenty-five cortical areas. J Comp Neurol 324:379-414.

Henderson Z, Finlay BL, Wikler KC (1988) Development of ganglion cell topography in ferret retina. J Neurosci 8:1194-1205.

Hestrin S (1992) Developmental regulation of NMDA receptormediated synaptic currents at a central synapse. Nature 357:686-689.

Kao C-Q, McHaffie JG, Meredith MA, Stein BE (1994) Functional development of a central visual map in cat. J Neurophysiol 72:266-272.

Keating MJ, Kennard C (1987) Visual experience and the maturation of the ipsilateral visuotectal projection in Xenopus laevis. Neuroscience 21:519-527.

King AJ (1993) A map of auditory space in the mammalian brain: neural computation and development. Exp Physiol 78:559-590.

King AJ, Carlile S (1993) Changes induced in the representation of auditory space in the superior colliculus by rearing ferrets with binocular eyelid suture. Exp Brain Res 94:444-455.

King AJ, Carlile S (1995) Neural coding for auditory space. In: The cognitive neurosciences (Gazzaniga MS, ed), pp 279-293. Cambridge, MA: MIT.

King AJ, Hutchings ME (1987) Spatial response properties of acoustically responsive neurons in the superior colliculus of the ferret: a map of auditory space. J Neurophysiol 57:596-624.

King AJ, Hutchings ME, Moore DR, Blakemore C (1988) Developmental plasticity in the visual and auditory representations in the mammalian superior colliculus. Nature 332:73-76.

King AJ, Jiang ZD, Moore DR (1998) Auditory brainstem projections to the ferret superior colliculus: anatomical contribution to the neural coding of sound azimuth. J Comp Neurol 390:342-365.

Knudsen EI (1982) Auditory and visual maps of space in the optic tectum of the owl. J Neurosci 2:1177-1194.

Knudsen EI, Brainard MS (1991) Visual instruction of the neural map of auditory space in the developing optic tectum. Science 253:85-87.

Knudsen EI, Knudsen PF (1983) Space-mapped auditory projections from the inferior colliculus to the optic tectum in the barn owl (Tyto alba). J Comp Neurol 218:187-196.

Knudsen EI, Esterly SD, du Lac S (1991) Stretched and upside-down maps of auditory space in the optic tectum of blind-reared owls: acoustic basis and behavioral correlates. J Neurosci 11:1727-1747.

Krug K, Smith AL, Thompson ID (1998) The development of topography in the hamster geniculo-cortical projection. J Neurosci 18:5766-5776.

Kudo M, Tashiro T, Higo S, Matsuyama T, Kawamura S (1984) Ascending projections from the nucleus of the brachium of the inferior colliculus in the cat. Exp Brain Res 54:203-211.

Lee PH, Helms MC, Augustine GJ, Hall WC (1997) Role of intrinsic circuitry in collicular sensorimotor integration. Proc Natl Acad Sci USA 94:13299-13304.

Linden DC, Guillery RW, Cucchiaro J (1981) The dorsal lateral geniculate of the normal ferret and its postnatal development. J Comp Neurol 203:189-211.

Meredith MA, Ramoa AS (1998) Intrinsic circuitry of the superior colliculus: pharmacophysiological identification of horizontally oriented inhibitory interneurons. J Neurophysiol 79:1597-1602.

Meredith MA, Stein BE (1986) Visual, auditory, and somatosensory convergence on cells in superior colliculus results in multisensory integration. J Neurophysiol 56:640-662.

Meredith MA, Stein BE (1990) The visuotopic component of the multisensory map in the deep laminae of the cat superior colliculus. J Neurosci 10:3727-3742.

Middlebrooks JC, Knudsen EI (1984) A neural code for auditory space in the cat's superior colliculus. J Neurosci 4:2621-2634.

Mooney RD, Klein BG, Jacquin MF, Rhoades RW (1984) Dendrites of deep layer, somatosensory superior colliculus neurons extend into the superficial laminae. Brain Res 324:361-365.

Mooney RD, Nikoletseas MM, Hess PR, Allen Z, Lewin AC, Rhoades RW (1988) The projection from the superficial to the deep layers of 
the superior colliculus: an intracellular horseradish peroxidase injection study in the hamster. J Neurosci 8:1384-1399.

Mooney RD, Huang X, Rhoades RW (1992) Functional influence of interlaminar connections in the hamster's superior colliculus. J Neurosci $12: 2417-2432$.

Moschovakis AK, Karabelas AB, Highstein SM (1988) Structurefunction relationships in the primate superior colliculus. I. Morphological classification of efferent neurons. J Neurophysiol 60:232-262.

Niida T, Stein BE, McHaffie JG (1997) Response properties of corticotectal and corticostriatal neurons in the posterior lateral suprasylvian cortex of the cat. J Neurosci 17:8550-8565.

Pallas SL, Finlay BL (1989) Conservation of receptive-field properties of superior colliculus cells after developmental rearrangements of retinal input. Vis Neurosci 2:121-135.

Pallas SL, Finlay BL (1991) Compensation for population size mismatches in the hamster retinotectal system: alterations in the organization of retinal projections. Vis Neurosci 6:271-281.

Perry VH, Linden R (1982) Evidence for dendritic competition in the developing retina. Nature 297:683-685.

Schnupp JWH, King AJ (1997) Coding for auditory space in the nucleus of the brachium of the inferior colliculus in the ferret. J Neurophysiol 78:2717-2731.

Schnupp JWH, King AJ, Smith AL, Thompson ID (1995) NMDAreceptor antagonists disrupt the formation of the auditory space map in the mammalian superior colliculus. J Neurosci 15:1516-1531.

Schnupp JWH, Giles JD, Baddeley R, King AJ (1997) Multisensory facilitation may be due to non-linearities in the neuron's rate-intensity function. Soc Neurosci Abstr 23:1542.

Shi J, Aamodt SM, Constantine-Paton M (1997) Temporal correlations between functional and molecular changes in NMDA receptors and GABA neurotransmission in the superior colliculus. J Neurosci 17:6264-6276.

Simon DK, O'Leary DDM (1992) Development of topographic order in the mammalian retinocollicular projection. J Neurosci 12:1212-1232.

Simon DK, Prusky GT, O'Leary DDM, Constantine-Paton M (1992)
$N$-methyl-D-aspartate receptor antagonists disrupt the formation of a mammalian neural map. Proc Natl Acad Sci USA 89:10593-10597.

Stein BE, Meredith MA (1993) The merging of the senses. Cambridge, MA: MIT.

Tay D, So K-F, Jen LS, Lau KC (1986) The postnatal development of the optic nerve in hamsters: an electron microscopic study. Dev Brain Res 30:268-273.

Thompson ID, Cordery PM (1994) The development of the retinocollicular map in the Syrian hamster. Soc Neurosci Abstr 20:1704.

Thompson ID, Cordery P, Holt CE (1995) Postnatal changes in the uncrossed retinal projection of pigmented and albino Syrian hamsters and the effects of monocular enucleation. J Comp Neurol 357:181-203.

Udin SB (1977) Rearrangements of the retinotectal projection in Rana pipiens after unilateral caudal half-tectum ablation. J Comp Neurol 173:561-582.

Wallace MN, Fredens K (1989) Relationship of afferent inputs to the lattice of high NADPH-diaphorase activity in the mouse superior colliculus. Exp Brain Res 78:435-445.

Wallace MT, Stein BE (1997) Development of multisensory neurons and multisensory integration in cat superior colliculus. J Neurosci 17:2429-2444.

Wallace MT, McHaffie JG, Stein BE (1997) Visual response properties and visuotopic representation in the newborn monkey superior colliculus. J Neurophysiol 78:2732-2741.

Wingate RJ, Fitzgibbon T, Thompson ID (1992) Lucifer yellow, retrograde tracers, and fractal analysis characterise adult ferret retinal ganglion cells. J Comp Neurol 323:449-474.

Withington DJ (1992) The effect of binocular eyelid suture on auditory responses in the guinea-pig superior colliculus. Neurosci Lett 136:153-156.

Withington-Wray DJ, Binns KE, Keating MJ (1990) The maturation of the superior collicular map of auditory space in the guinea pig is disrupted by developmental visual deprivation. Eur J Neurosci 2:682-692.

Wu GY, Malinow R, Cline HT (1996) Maturation of a central glutamatergic synapse. Science 274:972-976. 